\title{
PROGRÈS :RÉCENTS DANS LA BIOLOGIE DE L'ABEILLE
}

\author{
PAR \\ Rémy CHA UVIN \\ Station de Recherches apicoles, Bures-sur-Yvette.
}

Le nombre des travaux sur la biologie de l'abeille augmente avec une rapidité considérable. Comme dans la revue que j'ai présentée dans le même journal en I956, je laisserai volontairement de côté un certain nombre de lacunes qui ont déjà été comblées ou le seront par certains de mes collaborateurs. Par exemple, je ne traiterai pas des produits de la ruche, qui feront l'objet de revues spéciales; LouvEaUx a d'ailleurs publié dans les "Annales de l'Abeille " une thèse magistrale sur le pollen, qui ne peut être résumée. Je traiterai seulement en quelques mots du butinage, que LECOMTE doit examiner ici même dans quelques mois; et très brièvement aussi des substances actives dans la ruche (antibiotiques, substance royale) auxquelles PAIN et LAvIE viennent de consacrer deux thèses, que l'on pourra lire sous peu dans ces mêmes Annales. Ultérieurement aussi, LOUVEAUX fera une revue sur la reproduction et le problème des races chez l'abeille; c'est pourquoi je ne m'étendrai pas non plus sur ces mêmes sujets.

Comme dans ma revue de 1956 , je suivrai ici, l'ordre des événements biologiques.

\section{ELLEAGE ET BIOLOGIE DE LA REINE}

La fausse reine. - Quand on enlève une reine, une des ouvrières est suivie par ses congénères qui forment une sorte de cour autour d'elle, comme si elle était la reine pendant quelque temps au moins. Cette ouvrière ne pond pas (sauf dans le cas d'A pis cerana cerana, l'abeille japonaise, qui n'élève de reine qu'au moment de l'essaimage seulement, mais donne très facilement des ouvrières pondeuses.) La cour de la fausse reine est plus réduite que celle de la véritable (voir plus loin); elle est elle-même âgée en moyenne d'une dizaine de jours, son abdomen est un peu plus gros et 
plus lisse que celui de ses congénères, et elle n'exécute aucun travail d'ouvrière. Il arrive qu'elle se maintienne près d'un mois ou même qu'elle soit remplacée par une autre (SAKaGami, I958).

Deux types de cellules royales. - Les cellules royales sont différentes suivant que la reine a disparu accidentellement ou par remplacement spontané de la part des ouvrières. Dans le premier cas, les abeilles construisent des cellules royales à partir de cellules d'ouvrières, en les élargissant et en formant en dehors de la surface du cadre la hernie caractéristique dirigée vers le bas qui termine toutes les cellules royales. Alors la base de la cellule reste polyédrique. Mais si la ruche élève spontanément une reine, alors la base de la cellule royale, qui n'est plus arrondie, repose sur un gros bloc de cire, se forme d'habitude sur les bords du cadre, et non plus vers le centre comme dans le premier cas. De plus, certaines larves sont logées dans de grandes cellules isolées au centre du rayon et baignent dans une grande quantité de gelée royale : il y a peut-être là une ébauche de sexués de remplacement comme chez les termites; en effet ces larves paraissent maintenues plus longtemps sur la gelée royale que ne le sont d'habitude les larves ordinaires d'ouvrières. Elles pourraient servir à fabriquer ultérieurement des reines si le jeune couvain vient à faire défaut (Darchen et Vuillaume, I959).

Mais en cas d'élevage spontané, il est souvent difficile de reconnaître s'il s'agit de supersédure ou d'essaimage. BUTLER (I958) soutient que la supersédure est bien plus répandue qu'on ne le croit, et il a souvent vu cohabiter dans la même colonie la vieille reine et la reine de supersédure. Il enlève la jeune reine et garde la vieille; alors, si on enlève une partie des abeilles de la colonie, la vieille est encore capable d'inhiber la formation des cellules royales. Mais si l'on rajoute au contraire un kilogramme d'abeilles, des cellules royales sont immédiatement construites. Ce qui laisse penser que la supersédure est attribuable à un déficit d'ectohormone royale (voir plus loin). Ia vieille reine peut en fournir suffisamment à un petit groupe d'abeilles, mais non à un groupe plus nombreux. Une expérience très curieuse consiste à attacher la reine au rayon avec un fil métallique de trois pouces de long; elle s'arrête aussitôt de pondre, les abeilles la lèchent désespérément et pourtant elles entreprennent aussitôt la construction de cellules royales. BUTLER attribue ces résultats à un déficit de l'ectohormone dans ces conditions : mais on n'en voit gùère la raison et l'on peut se demander ce qui arriverait si le fil était plus long?

D'après VAGT (1955) on ne peut obtenir d'élevage royal avec des larves vieilles de plus de trois jours et les reines parfaitement normales proviennent seulement des larves les plus jeunes. Toutefois il n'existe pas de virage très net dépendant de l'âge larvaire et à partir duquel on 
puisse dire que la reine sera anormale. A vrai dire, les observations de DARChen et Vuillaume sur les pré-reines ou sexués de remplacement reposent tout le problème de l'âge des larves au moment de l'élevage royal.

SHiniarra (I953) pèse des cellules royales et leur contenu, larves ou gelée. Il y trouve très peu de gelée (45-I0o $\mathrm{mg}$ ) quand on enlève le couvain ouvert en ne laissant que les cellules d'élevage. Mais la quantité de gelée déposée augmente quand on redonne le couvain ouvert; et plus encore lorsqu' on laisse les abeilles pratiquer dessus un élevage spontané, sans cellules artificielles $(236-565 \mathrm{mg})$. On peut obtenir près de $800 \mathrm{mg}$ de gelée par cellule en se bornant à enlever la reine sans toucher autrement à la ruche, les abeilles étant laissées libres d'élever quand et où elles l'entendent. Mais 1e poids des larves royales est bien plus variable et ici les rapports avec les perturbations expérimentales sont beaucoup moins clairs.

L'élevage artificiel. - Orosi PAL (1958) remarque, en accord avec VUm, AUme, que les abeilles acceptent mieux les cupules de cire avec une larve greffée à l'intérieur que les cellules d'ouvrières contenant du jeune couvain et placées l'ouverture vers le bas. Il a essayé également l'élevage à partir de larves d'âge croissant de I-2 jours à 9 -Io jours. L,e maximum des réussites correspond à I-2 jours. On peut aussi, à l'aide d'un petit emporte-pièce greffer l'œuf, ainsi que le petit disque de cire sur lequel il repose, ce qui assure le maximum de sécurité aux manipulations. II semble bien que les reines obtenues à partir d'œufs soient plus grosses que celles qui proviennent des greffes de larves. Vuillaume (I958) a pu vérifier que la substance qui dans la propolis inhibe l'acceptation des cellules royales peut s'en extraire par la vapeur après saponification. Les cupules peuvent encore être acceptées quand elles ne dépassent que de peu la taille normale. Alors les ouvrières savent les rétrécir par une cloison partielle et mener à bien l'élevage royal (Vuillaume, I957). Mais les larves déposées dans des cupules ou des barquettes de cire beaucoup trop larges, même cloisonnées au préalable, ne sont pas acceptées. C'est alors la forme du fond et la juxtaposition des bords des cloisons qui perturbe les ouvrières. Si en effet une cellule royale peut subir au cours de son étirage des modifications considérables, sa base n'est jamais remaniée; les ouvrières se contentent de la refuser en bloc si la base ne convient pas (Vuillaume i958). Dans l'élevage artificiel, on peut sans inconvénients supprimer le starter (colonie d'abeilles orpheline où débute l'élevage) et introduire directement les larves dans le finisseur (colonie où la reine est bloquée sur deux ou trois cadres au moyen d'une grille à reines ; les larves sont greffées dans la partie orpheline). Mais il faut alors déposer au fond des cellules une quantité assez notable de gelée royale (Vuir, AUmE, I958). 
Il est assez curieux de noter que les ouvrières peuvent démolir des cellules royales dont la reine est sortie pendant qu'à côté d'elles d'autres ouvrières approvisionnent une nouvelle série de jeunes cellules au début de leur évolution (Vuiliaume, I959).

\section{La reine et sa cour. La reine dans la ruche.}

L'activité d'une reine affecte le nombre d'ouvrières qui compose sa cour et le nombre de celles qui la lèchent. La cour se compose en moyenne de six abeilles en été lorsque la reine se promène sur les rayons, de 8 quand elle pond, de ro quand elle ne bouge pas. On ne voit pas de changement appréciable dans le nombre de ses accompagnatrices au cours de la préparation à l'essaimage. L,es abeilles en hiver ne manifestent que peu d'intérêt pour la reine et ne la lèchent que rarement, ce qui donne à penser que la substance royale n'est pas toujours très active ; le léchage pourrait correspondre aussi à des mouvements de nettoyage (Putzbewegungen) si fréquents chez les insectes et qui se développeraient plus ou moins. Vis-à-vis de la reine, les ouvrières pratiquent aussi des «monvements de seconage " assez énigmatiques. Ces mouvements s'observent aussi entre ouvrières : alors une abeille en agrippe une autre avec ses pattes antérieures et les deux corps s'agitent en vibrant dans le plan vertical pendant une seconde environ; parfois l'abeille se sert des autres pattes ou se borne à mantenir sa tête contre le corps de l'autre abeille pendant qu'elle fait vibrer son propre corps. La reine est rarement secouée de la sorte au printemps, mais elle l'est de plus en plus au fur et à mesure que l'essaimage approche ; elle l'est encore quand elle commence à pondre après la fécondation; mais peu après le nombre des phases de secouage diminue rapidement. Il s'agit sans doute ici de ce que MiLum appelait la D.VAV. I'âge des abeilles secoueuses est extrêmement variable, de 3 jours à plus d'un mois (ALLEN, I957, I959).

Je ne puis résumer ici les très importants travaux de PAIN sur l'atlractivité des reines d'abeille. Rappelons seulement la découverte récente, faite en même temps par BuTLER et PAIN d'une substance émise par la reine qui inhibe la construction des cellules royales (BUTLER) et le développement des ovaires des ouvrières (PAIN) (queen substance, ectohormone, phérormone). Ces travaux ont renouvelé nos connaissances sur la physiologie de la ruche en tant que corps social. Il semble exister de curieux rapports entre les antibiotiques que l'on peut extraire du corps même des ouvrières et de la reine ( $\mathrm{I}_{\mathrm{AVIE}}$ ) et la substance royale.

I'attractivité des reines vierges est en liaison directe avec le nombre d'ouvrières qui les accompagnent dans la cagette. Toutefois même chez les reines élevées absolument seules, une certaine attractivité se manifeste au bout de quelques jours. D'ailleurs, non seulement 1'attractivité des reines par rapport aux ouvrières est très variable, mais encore 
l'ectohormone ne paraît émise chez la même reine que d'une manière irrégulière (PAIN, I956, I959). C'est la glande mandibulaire de la reine qui attire fortement les ouvrières. Eille est en même temps fortement antibiotique ; la substance attractive et antibiotique apparaît dans la glande du $5^{\mathrm{e}}$ au $8^{\mathrm{e}}$ jour, et plus tôt lorsque les reines sont en contact avec les ouvrières. D'autre part, comme LAVIE l'a montré, les ouvrières sécrètent une substance antibiotique qui est répandue sur le tégument. Or, mieux les ovaires sont développés chez les ouvrières et plus on trouve d'antibiotique sur leur corps. En présence de la reine, le taux d'antibiotique diminue et les ovaires se réduisent, alors que chez la reine la quantité d'ectohormone augmente (LAvie et Pain, I959).

Le poids des reines constitue une variable importante qui peut être modifiée par exemple au cours d'un maintien à l'étuve en présence des ouvrières (KHARCHEva, I957). Si l'on conserve en cagette de I7 à 46 jours la reine féconde qu'on vient d'enlever d'une colonie, en présence de Io ouvrières que l'on renouvelle au fur et à mesure des décès, le poids de la reine passe de 230 à $\mathrm{I} 68 \mathrm{mg}$ par exemple. Lorsqu'on introduit dans 1a cagette des abeilles supplémentaires, le poids augmente temporairement. D'ailleurs, le poids des reines vierges introduites dans les colonies augmente très sensiblement. Notons d'après MILOJEvic qu'il paraît possible de conserver en cagette et pendant très longtemps ( $\mathrm{I}-7 \mathrm{mois}$ ) des reines fécondées en présence d'un petit nombre d'abeilles à l'étuve, ou, mieux, à la température du laboratoire (vers $\left.15^{\circ}\right)$. Ces mêmes reines, remises dans une colonie pourraient pondre à nouveau très normalement. Skrobal (I958) ne trouve pas de rapport entre le poids des reines et la vigueur de l'essaim ou sa rapidité de développement. Le poids des reines fécondées dépasse de 34 à $75 \mathrm{p}$. roo celui des non fécondées ; par ailleurs le poids des reines augmente durant le printemps et l'été puis décroît. Le poids maximum des ouvrières est atteint en maijuin, le minimum en automne.

I1 faut rappeler ici d'anciennes recherches de Komarov et Alpatov (1934) qui remarquent chez les reines fécondées une augmentation de poids de $30 \mathrm{mg}$ par rapport aux reines vierges ce qui s'explique sans doute par l'augmentation rapide du poids des ovaires après la fécondation. D'autre part, certaines différences d'ordre racial ont pu être mises en évidence : ainsi les reines bavaroises sont en moyenne de 20 à $25 \mathrm{mg}$ plus légères que celles de Russie centrale. ZANDER a prétendu que les reines d'élevage étaient plus légères, mais la statistique ne confirme pas une telle différence. En général les reines méridionales sont plus légères que les reines du Nord, ce qui est à rapprocher de la diminution de taille des ouvrières quand on se déplace vers le Sud. On a mesuré également les dimensions de l'ovaire après l'avoir fixé, photographié et en avoir suivi les contours au curvimètre ; on a compté également le nombre de follicules 
ovariques chez les abeilles de Tula, de Mingrélie, d'Allemagne et d'Amérique. La race mingrélienne (nord du Caucase) comptait jusqu'à 369 follicules pour les deux ovaires, et les races allemandes 320. Les ovaires des races de la Russie Centrale sont plus gros encore que ceux des Mingréliennes; mais on ne trouve pas de corrélation entre le poids du corps et le nombre des follicules.

Les modifications qui surviennent lorsque la reine avance en $\hat{a} g e$ ont été étudiées par FYG (I956). Chez la jeune reine fraîchement éclose, le rectum contient toujours un méconium huileux et rougeâtre, les papilles rectales sont transparentes et incolores avec une bordure jaune. Mais après un an la bordure brunit et ne cessera plus de foncer avec l'âge. La glande à venin est bien développée chez les jeunes, mais commence à brunir et à dégénérer dès les premiers mois ; les tubes de Malpighi brunissent aussi avec l'âge alors qu'ils sont incolores chez la jeune reine. Leur teinte est jaunâtre la première année, jaune ou jaune vert la seconde, verdâtre 1a troisième et brune ensuite. La couleur des œenocytes fonce également comme l'avait déjà remarqué Koschevnikov. Incolores chez la jeune reine, il s'y développe au bout des premiers mois des grains jaunâtres qui brunissent au cours de la deuxième ou troisième année. FYG considère comme spécialement intéressant pour l'estimation de l'âge le durcissement d'une valvule de 1'appareil génital, la valvula vaginalis.

\section{LA FÉCONDATION ET LA PONTE}

\section{Le vol nuptial.}

Pour la préparation des nuclei de fécondation Triasko (I95I) donne une technique qui permet une économie maximum du nombre d'abeilles à employer. Il conserve les cellules royales prêtes à éclore et les reines qui en sortent dans de petites cages avec 5-7 abeilles de 2-3 jours, en leur fournissant une nourriture dont la proportion doit être exactement déterminée ( $\mathrm{I}-2$ partie de gelée royale dans 50 parties de miel). Lorsque les reines atteignent l'âge de 8-ro jours, on les transfère dans un micronucléus de I5 $\times 4 \times 4 \mathrm{~cm}$ avec IO-I 5 abeilles de $2-3$ jours. Une partie de ce micronucléus contient un fragment de rayon, l'autre un nourrisseur empli du mélange ci-dessus. On range les cagettes dans une hausse à plancher grillagé au-dessus d'une forte colonie qui leur donne sa chaleur. On ne laisse sortir les reines qu'à l'âge de IO-I2 jours et seulement de 2 à 5 heures de l'après-midi.

Sil'on en croit RUTTNER (I957) l'instinct de reproduction chez les reines n'est pas automatique, il est induit par les butineuses qui manifestent à leur égard un comportement agressif spécial. D'ailleurs, d'après ProsT (r958) on peut constater à la suite d'un élevage maternel naturel ou pro- 
voqué, la présence d'une reine libre qui tolère les cellules royales operculées. Les jeunes reines restent alors dans leurs cellules à l'opercule fort ébréché dont cependant elles pourraient sortir : la preuve en est qu'elles en sortent effectivement en quelques minutes quand on enlève la reine déjà libre. N'existerait-il point une séquestration temporaire des reines tant que la première n'est pas sortie?

Dans les conditions normales, les ouvrières ne tiennent aucun compte de la reine qui vient d'éclore. Mais dès le milieu de la première journée de sa vie, l'intérêt des abeilles à son égard devient très vif : elles la palpent, la lèchent, la nourrissent et la secouent parfois (v. plus haut observations d'ALLEN sur le secouage). Il arrive d'ailleurs que la reine soit véritablement attaquée et bousculée; elle s'enfuit alors en émettant un son aigre, qui semble "frapper de stupeur" les abeilles. Mais les poursuites deviennent acharnées; alors la reine en fuyant finit par trouver le trou de vol et part se faire féconder. Si la reine est traitée avec douceur par les ouvrières, c'est que son essor va être retardé ou n'aura pas lieu. Mais chez les colonies qui attaquent vigoureusement la jeune reine se trouvent souvent des ouvrières pondeuses; on a pu observer toutefois des colonies privées de pollen et dont les ouvrières n'avaient que de petits ovaires, attaquer vigoureusement leur reine (HAMmaN, I957).

Lorsque les abeilles s'envolent, elles montent rapidement à Io ou I 2 mètres et $\mathrm{y}$ restent en moyenne. Mais les mâles et les reines volent nettement au-dessus de cette couche, et surtout les reines, qui volent plus haut encore que les mâles; au cours de leurs sorties successives, elles abaissent chaque fois leur niveau de vol jusqu'à ce qu'elles rejoignent le niveau de vol des mâles. On remarquera d'ailleurs que si les ouvrières entrent souvent dans les maisons, on n'y a jamais vu de mâles, qui volent trop haut (Nol,D, I956). A vrai dire, je me demande si le problème du vol nuptial ne devrait pas être reconsidéré à nouveau à la lueur de ce qu'on sait des "bals d'abeilles" - ou de ce qu'on croit en savoir! Il semble exister, dans certaines zones bien déterminées, des lieux d'accouplement spécialisés, où les mâles arrivent à couvrir même une reine maintenue dans la main de l'expérimentateur. Certains de ces bals d'abeilles seraient connus des apiculteurs depuis près de $5^{\circ}$ ans, et on y entend d'habitude un intense bourdonnement. Si le fait est bien exact, on y pourrait trouver une des particularités les plus curieuses de la biologie des abeilles, rappelant quelque peu ce que l'on sait des chemins de mâles chez les bourdons, chemins que suivent les femelles qui veulent se faire féconder. Cela expliquerait aussi l'insuccès des très nombreuses expériences de fécondation en serre comme celles de BöTTCHER ; sans doute ne se trouvaientelles point sur un emplacement où les mâles acceptent de copuler?

D'après MACKENSEN (I95I) les femelles ont tendance à copuler en dehors de leur propre ruche et par conséquent à éviter l'inbreeding qui 
apporte des gênes léthaux et diminue la vitalité du couvain. On sait d'ailleurs qu'on a tenté d'appliquer aux abeilles les techniques d'hétérosis qui ont donné dans le cas du mais des résultats si intéressants. Pour cela deux colonies sont amenées par inbreeding à une dégénérescence rapide et on croise leurs descendants mâles et femelles. I,es reines hybrides d'hétérosis donnent beaucoup plus d'œufs et souvent plus de miel que les témoins (CALE, I956). Si l'observation de MACKENSEN est exacte il est alors étrange de voir les reines éviter spontanément l'inbreeding ; en tout cas, d'après le même auteur, la reine, sortant de la ruche fait de grands cercles autour du rucher de fécondation et il arrive alors qu'elle soit suivie par les mâles de sa propre ruche. Puis elle pique droit devant elle (vers les bals d'abeilles?) et alors les mâles de sa ruche et de son rucher l'abandonnent.

L,es rapports de la météorologie et du vol nuptial ont été étudiés. notamment par Soczek (I958). I,es reines peuvent voler sans s'accoupler entre II et I7 heures. L.es vols suivis d'accouplement se produisent surtout de I4 h I5 à I5 h I5, et les mâles volent au même moment. Le vol. nuptial s'observe de $\mathbf{r} 9$ à $3^{\circ}$ pour un vent de $\mathrm{I}-9 \mathrm{~m} / \mathrm{sec}$. Les nuages n'ont pas d'influence sur le vol de la reine; mais ils gênent ou empêchent celui des bourdons. L'accouplement effectif survient de 20 à $28^{\circ}$ pour un vent de $\mathrm{I}-8 \mathrm{~m} / \mathrm{sec}$. La durée des vols de reine non suivis d'accouplement varie de 5 à Io minutes entre $I 9^{\circ}$ et $28^{\circ}$. Les vols avec accouplement durent de II à I7 minutes; la température est alors sans influence sur la durée du vol. D'autre part, la plupart des reines (sur 243 reines observées) vont voler 6-I3 jours après 1'éclosion et s'accouplent Io à r2 jours après. Quelques reines commencent à voler le premier jour et certaines seulement le $28^{\mathrm{e}}$ jour. La plupart vont s'accoupler le $4^{\mathrm{e}}$ jour. Le vol d'accouplement est généralement le second (SOczEK, I958) (voir plus haut les travaux de RU'TTNER).

La ponte. Parmi les II2 colonies observées par MEDVEDEv (I956) la reine fécondée commence à pondre à 7 -r 9 jours. Ce sont les reines des essaims et les reines obtenues par supersédure qui pondent le plus tôt, puis viennent les reines introduites dans les colonies orphelines; en dernier lieu enfin, celles qu'on introduit dans des nucléi, dont la population est forcément assez réduite. RobInson (I95I) a pu réduire le laps de temps avant l'oviposition par des secousses électriques (optimum 5 milliampères pendant 30 secondes). Il se rapproche ainsi de l'accélération du début de la ponte obtenue par MACKENSEN (I947) en traitant les reines par le gaz carbonique (Io minutes dans le gaz pur deux fois, à 24 heures d'intervalle, sont nécessaires). Le phénomène du secouage signalé par ALLEN (v. plus haut) se retrouve souvent avant le dépôt du premier œuf dans les cellules d'ouvrières (ALLEN I958).

Différentes anomalies de la ponte ont été signalées par FyG (1957). 
Rappelons qu'une reine vierge qui vient de pondre des cufs de mâles ne peut plus s'accoupler, mais on peut toujours l'inséminer artificiellement. Une vieille reine pondra aussi des œufs de mâles. Sa spermathèque n'est pas forcément vide, mais les spermatozoïdes y sont dégénérés, immobiles et enroulés (Ringelsamen). Même une jeune reiné en bonne santé pondra éventuellement des cufs de mâles si sa provision de sperme s'est trouvée incomplète pour une raison ou pour une autre; souvent aussi une reine qui vient de s'accoupler pond au début des œufs de mâles, mais cesse bientôt. Mais une observation capitale de FYG montre que la principale cause de la ponte anormale d'xufs de mâles est une maladie à virus qui pénètre dans l'organisme de la reine à travers l'intestin. Le virus provoque des modifications caractéristiques des noyaux dans les organes génitaux. On en trouve d'ailleurs aussi bien chez les reines vierges que chez les reines fécondées; ce qui induit FYG à repousser l'opinion de BAUMGARTNER suivant laquelle la maladie serait transmise par les mâles; on ne voit d'ailleurs pas trace des lésions caractéristiques chez les ouvrières ni les mâles. Une cause plus rare de la ponte anormale d'œufs de mâles consiste dans la dégénérescence des glandes accessoires de la spermathèque, l'empoisonnement par divers toxiques ou l'emploi de rayons avec cellules trop grandes (mais la ponte peut se normaliser même dans ces conditions, voir plus loin).

Il existe aussi des reines dont les outs n'éclosent pas, qu'ils soient fécondés ou non; cependant leur développement embryonnaire progresse parfois fort loin; et on ne trouve chez la mère aucune maladie décelable. Il doit donc s'agir d'une anomalie d'ordre génétique ( $\mathrm{HITCH}^{\prime}$ COCK, I956). Filanders (I959) a trouvé dans le sud des Etats-Unis une souche de reines dont les œufs n'éclosent que rarement; dans ce cas les jeunes larves des mâles vivent un peu plus longtemps que celles des ouvrières, mais ne tardent pas à périr néanmoins. Ces reines paraissaient normales et leur spermathèque contenait la quantité normale de spermatozoïdes. Mais comme le remarque Franders (r957) chez la reine ainsi que chez beaucoup d'Hyménoptères, les œufs non pondus dégénèrent dans les ovaires et sont plus ou moins réabsorbés. Il n'en reste qu'un anneau jaunâtre, à la base de l'ovaire des vieilles reines. Il est possible qu'une rétention partielle amène une résorption partielle, les œufs pouvant alors être pondus sans être viables.

Le déterminisme $d u$ sexe présente chez l'abeille encore bien des obscurités. TUCKER (I958) a étudié la ponte de nombreuses reines vierges confinées dans des nuclei. Il est bien vrai qu'elles donnent surtout des mâles, mais aussi quelques ouvrières et plus rarement des gynandromorphes; mais la proportion d'ouvrières, habituellement de I p. Ioo peut s'élever jusqu'à 7 p. Ioo ; elle s'élève surtout lorsque l'oviposition vient de commencer ou après une interruption temporaire de la ponte, au 
moment de la reprise. Ces ouvrières " automictiques " se rencontrent dans toutes les races, mais certaines en produisent davantage, par exemple 1a fameuse abeille du Cap que tout le monde cite, mais que personne n'a étudiée depuis le début du siècle. Mackensen (1943) a mis en évidence dans la race caucasienne que 3 reines sur 13 pouvaient, sans fécondation, fournir des ouvrières; et sur deux souches d'italiennes, I 8 sur 4 I le pouvaient également. Comme le fait remarquer Rothenbuhler (I957) il semble que comme chez l'Hyménoptère Habrobracon les hétérozygotes soient femelles, les haploïdes mâles et les homozygotes mâles diploïdes à peu près inviables. Chez Apis indica la femelle serait tétraploïde et le mâle numériquement haploïde mais génétiquement diploïde (par suite de l'existence de chromosomes couplés) (Deodikar, Thakar, Shafr, I959). D'autre part, les souches à gynandromorphes se perpétuent par la reine, et les mâles de ces souches ne transmettent pas cette particularité à leur descendance. Mais si d'autre part les reines à gynandromorphes sont croisées avec les mâles de la souche normale, la gynandromorphie disparaît peu à peu (ROTHENBUHLER, I955).

L'observation journalière de la ponte de la reine, par une technique appropriée (ChaUvin, I956) permet de mettre en évidence la grande indépendance de ce phénomène par rapport aux conditions extérieures. Seule la carence alimentaire poussée assez loin peut arrêter la ponte et dans ce cas l'apport de glucides à la colonie provoque un nouveau départ. Mais si on ajoute aux sucres du pollen ou de la gelée royale qui fournissent de l'azote et peut-être aussi d'autres substances favorisantes, l'augmentation de la ponte atteint le double de celle des colonies témoins nourries au sucre. Il existe, enfin un arrêt spontané automnal que même le renforcement de l'alimentation ne peut briser et que l'on doit peut-être attribuer à une diapause.

\section{LES MALES}

La naissance des mâles. - On ne sait que bien peu de choses sur le déterminisme de la formation des cellules de mâles. Seuls Darchen et VuILlaume ont exécuté là-dessus des travaux récents. La reine inhibe complètement la construction des cellules de mâles dans les colonies de ro 000 à 15000 abeilles. La présence de jeune couvain naissant bloque à son tour la construction des cellules d'ouvrières (DARCHEN et VUILLAUME: 1958). Lorsqu'on introduit une jeune reine fécondée dans une ruche garnie uniquement de cellules de mâles, une très grande partie des alvéoles sont transformées en cellules d'ouvrières par raccourcissement de la partie la plus externe de la cellule, avec élargissement des bords. Il se forme ainsi un anneau épais, sorte de bourrelet, autour de l'orifice de la cellule. Au moment de la nymphose, les cellules sont fermées par un 
petit opercule bombé qui permet d'allonger quelque peu la cellule et d'y loger la tête de la nymphe; ces modifications surviennent avant que la reine ait pondu (DARCHEN, VIZIER, VUILLAUME I957). Notons à ce propos une curieuse observation d'IGNATOVIcH (I953) : les abeilles élevées sur cellules agrandies n'entreprendraient que très rarement la construction de cellules de mâles.

Si de très nombreux auteurs ont tenté de provoquer un accroissement de la taille des ouvrières en les faisant élever dans des cellules élargies, VoLOSEVICH (I956) est le seul à ma connaissance à avoir tenté la même expérience pour les cellules de mâles; les cellules élargies avaient dans ce cas $7,39 \mathrm{~mm}$ de diamètre. Les mâles qui en proviennent pèsent I8 p. Ioo de plus que les normaux; la longueur et la largeur du front sont respectivement augmentées de 5 et 7 p. roo.

KuLshinskinata (I955) obtient un excès de poids plus net encore de 22,7 p. Ioo par rapport aux mâles normaux; la longueur des ailes et des sternites est notoirement plus grande.

ALLEN (1958) a compté le nombre de cellules de mâles operculées dans plusieurs colonies par rapport à la quantité totale de couvain. Le couvain mâle n'est guère présent que pendant la moitié de la période d'élevage. On trouve d'ailleurs de grosses différences dans le nombre des cellules mâles suivant les colonies, sans rapport précis avec le nombre des cellules royales ni la préparation à l'essaimage. Il semble toutefois qu'en gros l'intervalle entre le maximum d'élevage des mâles et le début de l'apparition des cellules royales est en moyenne de trois semaines.

\section{La nourriture des mâles.}

Les larves de mâles sont nourries de 5 à I4 fois par heure, le temps de distribution étant en général de 33 secondes ; mais, comme dans le cas des larves d'ouvrières, on peut noter des visites non suivies de distribution de nourriture. La fréquence des visites augmente avec l'âge des larves : 776 visites en 24 heures à l'âge de $\mathrm{I}$ jour ; 1507 à 6 jours. Les mâles adultes d'un jour sont nourris 25 fois par heure, ceux de 2 jours $I 6$ fois. Jusqu'à 1'âge de 4 jours, les mâles sont nourris entièrement par les ouvrières, mais les mâles plus âgés peuvent prélever eux-mêmes le miel dans les rayons. On peut estimer que pour élever I 000 mâles, de l'œuf au vol de l'adulte, $6,89 \mathrm{~kg}$ de miel doivent être dépensés (LEvEnETs, 1956). D'après FrEE (I957) les mâles, qui passent la plus grande partie de leur temps sans bouger dans la ruche sont nourris la plupart du temps par les jeunes nourrices de 2-4 jours bien que les nourrices plus âgées puissent le faire à l'occasion. L,e sperme est à $\mathrm{pH} 6,8-7, \mathrm{I}$, parfois seulement à 6 . I1 peut rester actif, même dans les solutions à $\mathrm{I}, 2 \mathrm{p}$. Ioo de chlorure de sodium. 
L'adjonction d'une solution alcaline de citrate de $\mathrm{Na}$ arrête presque complètement sa motilité ; une dilution avec de l'eau provoque rapidement la mort. Mais on peut dessécher les spermatozoïdes et les faire revivre à condition de les remettre dans la solution saline juste au moment où s'arrêtent les mouvements. Le sperme vit très bien dans la solution glucosée utilisée pour l'insémination expérimentale des mammifères, et peut revivre après un refroidissement rapide de la solution $\grave{a} o^{\circ}$; on peut donc le conserver, comme on conserve le sperme des mammifères domestiques (SMrrnov, I953).

\section{L'expulsion des mâles.}

Chez les Caucasiennes, les mâles résident surtout sur les cadres extérieurs, mais chez les Italiennes on les trouve partout et ils ne sont refoulés sur les cadres extérieurs que peu avant leur expulsion. A ce moment, et chez les deux races, les abeilles les repoussent sur les parois, puis sur le plancher, puis au dehors. Si la miellée cesse provisoirement, les Caucasiennes peuvent expulser les mâles dès le mois de juin. Mais chez la plupart des colonies cela commence au milieu de juillet et dure jusqu'à la mi-août; les Italiennes dans les mêmes conditions n'expulsent que un mois et demi plus tard. Dans la même colonie, alors que certaines ouvrières expulsent les mâles, d'autres les nourrissent ; on observe donc de grandes différences individuelles dans le comportement. Les abeilles qui attaquent les mâles sont probablement des butineuses sans emploi. Notons de plus que les nourrices continuent souvent à élever le couvain de mâles pendant que leurs congénères expulsent les adultes (LEVENETS, I956; FREE, I957).

\section{LE DEVELOPPEMENT DES LARVES}

Comme je le disais dans une revue précédente, un certain nombre d'auteurs ont recherché des méthodes de mesure du développement de l'élevage. Une des techniques les plus anciennes et peut-être la plus pratique est constituée par la photographie des cadres à travers un treillis après en avoir secoué les ouvrières (ARMBRUSTER, I924-25). Avec beaucoup de temps et de peine, et aussi au prix d'un grand trouble dans la vie de la ruche on peut obtenir de bonnes courbes de développement du couvain. On a essayé aussi de compter le nombre de cadres de couvain. Mais il faut se rappeler que des fuctuations considérables de la quantité de couvain présente peuvent se produire sans que le nombre des cadres porteurs de couvain en soit affecté (SIMPSON, I958). JEFFREE (I958) a essayé de mesurer la surface de couvain avec plus de précision en posant dessus un cadre métallique porteur d'ellipses et de lignes diverses 
qui permettent un certain nombre de corrections. Mais il faut bien convenir que la méthode de mesure du développement de la colonie qui ne la perturberait pas gravement est encore à découvrir. Je m'en suis approché avec la ruche à une face, aux cadres collés sur une vitre et qui permettait de compter par transparence les œufs au fur et à mesure de la ponte (Chauvin, I950). Mais ce résultat n'est atteint qu'au prix de grosses modifications dans la structure de la grappe, qui doivent retentir sur le développement de la colonie. Quoiqu'il en soit, AlLEN et JEFFreE (I956) trouvent que le développement est en rapport avec la quantité de pollen emmagasiné, mais aussi et surtout avec le nombre d'abeilles dans la colonie. UCHIDA et SAKAGAMr (I955) démontrent, par des courbes très nettes que les cellules royales semblent être édifiées surtout quand le rapport larves/nourrices passe par un minimum.

L'influence du nourrissement sur le couvain a fait 1'objet des études de Podoba (I955) : si l'on administre par jour aux colonies $300 \mathrm{cc}$ de sirop plus I g par litre de thy roïdine le développement larvaire est raccourci de deux jours et celui des nymphes de deux jours également ; les colonies se développent nettement plus vite. En mélangeant au sirop un extrait de nymphes de mâles le développement larvaire est raccourci d'un jour et demi, mais pas le développement nymphal (?). L'hétéroauxine à Io $\mathrm{mg}$ par litre raccourcirait également d'un jour le développement larvaire. Cette substance stimulerait aussi la ponte, mais en provoquant la supersédure.

L'élevage in vitro. - Il est certain que le seul moyen précis d'étudier le développement des larves consiste à les élever in vitro, à l'étuva et à l'abri des ouvrières, ce qui n'est pas aussi difficile qu'on pourrait le croire. On connaît là-dessus les anciens travaux de von RHEIN qui ont été repris par Michaei, et Abramowitz (I955), Hoffman (I956), Weaver (I957), SснміDт (I959). I a conclusion la plus nette qu'on en peut dégager est que seule la gelée royale est capable d'induire un développement normal. Mais très souvent les " reinəs " ainsi obtenues sont plutôt des formes de transition (ouvrières à gros ovaires) telles qu'on en rencontre souvant dans les ruches. WEAVER soutient que les raines typiques ne sont obtenues que par l'emploi de geléa extrêmement fraîche retirée toutes les deux heures de la ruche. Il semble être suivi par Smrth qui utilise de la gelée prélevée chaqua jour dans les ruches, et correspondant à l'âge de la larve ; cet auteur a pu élever ainsi 25 reines sur I03 larves ; certaines ont été fécondées et ont pondu. Le développement ne s'accomplit pas sur de la gelée prélevée dans les cellules d'ouvrières.

Les transferts de larves. - Ces expériences, qui ne sont point sans rapport avec le déterminisme du sexe, posent de curieux problèmes. Volosevich (I958) transfère des larves d'ouvrières dans des cellules de 
mâles dont la larve a été enlevée et vice-versa mais en laissant la nourriture qui se trouve au fond de la cellule. Alors sur 290 larves d'ouvrières transplantées, I32 cellules operculées donnent 97 ouvrières et 3 I mâles. Dans une autre expérience sur I6I larves d'ouvrières on obtient Io6 ouvrières et ro mâles. Sur I 54 larves de mâles greffées dans des cellules d'ouvrières, on obtient 44 mâles et 6 ouvrières. WEAvER (I957) utilise une technique un peu différente : il transporte dans des cellules d'ouvrières des larves qui ont fait un certain stage dans les cellules royales. Il est alors possible d'obtenir des ouvrières normales quand la permutation est réalisée après I jour seulement passé dans les cellules royales. Si les larves ont été portées dans les cellules royales à l'âge de $2-3$ jours et qu'on les y laisse I jour, les adultes tendent à prendre certains caractères royaux. Il en est de même si on les laisse deux jours au lieu d'un seul dans les cellules royales. Notons qu'après le transfert cellules royales dans cellules d'ouvrières peu de larves sont acceptées par les nourrices, et, de plus, beaucoup meurent. Inversement, si l'on greffe dans les cellules royales des larves d'ouvrières de plus en plus âgées, des reines normales ne sont obtenues que jusqu'à l'âge de deux jours. Avec les larves de trois jours, certains individus deviennent encore des reines typiques, mais beaucoup sont anormaux et ressemblent à des ouvrières. Avec des larves de 4 jours WEAVER n'a obtenu qu'un adulte, qui ressemblait à une ouvrière, avec toutefois certains caractères de reine. Par ailleurs, si on refroidit entre 8 et $12^{\circ}$ un cadre de couvain et si on le replace ensuite dans la ruche, les quelques larves qui réussissent à survivre donnent des gynandromorphes (Rosch et Khalifman, in Smith, I959).

D'autre part, HAYDAK (1958) est porté à croire que dans les colonies pourvues d'une reine, les nourrices reconnaissent le sexe des larves; car même si on transfère des larves d'ourrières dans les cellules de mâles, la nourriture qui leur est distribuée se rapproche plus de celle des ouvrières que de celle des mâles. Mais dans les colonies depuis longtemps sans reine, il semble que les nourrices ne distinguent plus si bien le genre de nourriture qu'il faut distribuer et donnent même de la gelée royale à des larves de mâles. Pourtant dans les cellules royales que peuvent faire ces colonies, la gelée semble normale.

\section{Les caractères somatiques et l'influence de l'élevage.}

Nous pénétrons ici dans un domaine assez singulier, qui a vivement intéressé les chercheurs russes. Je rappellerai d'abord les études déjà anciennes de Michailof (I93I) : si 1'on modifie les conditions d'élevage des larves en diminuant par exemple le poids de l'essaim, on ne voit guère de changement dans les proportions somatiques, sauf si l'essaim est très faible: alors les abeilles filles sont un peu plus petites. Lorsqu'on 
coupe en deux un rayon et qu'on en laisse une moitié dans les conditions normales tandis que l'autre est placée à $35^{\circ}$, sans abeilles, pendant une dizaine d'heures ( 10 p. Ioo de la vie larvaire) les ailes et la trompe sont nettement plus petite chez les abeilles qui éclosent de la moitié de cadre ainsi traitée. D'autre part, il existe des colonies où la taille moyenne est plus grande. On peut faire élever par de grosses abeilles un rayon de couvain de petites et vice-versa. I a trompe et les ailes s'allongent alors chez les petites abeilles ainsi élevées, mais non l'abdomen. Les grosses abeilles diminuent quand elles sont élevées par des petites. Les variations les plus grandes sont obtenues, dans le sens de l'allongement de la trompe, par une haute température d'élevage du couvain. Pour le nombre des crochets alaires, par l'élevage en rayons neufs au lieu de l'élevage en rayons encombrés de vieux cocons; dans le sens de l'accroissement de la longueur de l'abdomen, pat une nourriture pauvre en pollen. SchinajeW (I952) greffe, suivant toutes sortes de combinaisons des reines dans des colonies de races différentes. I.es premières abeilles qui éclosent, venant des reines, prennent dans une certaine mesure les caractères morphologiques des nourrices. Gubin et KHALIFMAN (I952) font élever du couvain de Caucasiennes dans une colonie d'abeilles du nord de la Russie. Ia colonie de Caucasiennes qui en résulte scelle son miel avec des opercules bombés comme ceux des abeilles du Nord et non plats comme ceux des Caucasiennes (certains opercules toutefois étaient de forme intermédiaire). I,e couvain d'abeilles du Nord élevé par les Caucasiennes présente aussi des modifications d'opercule. I a longueur de la langue est de 7,288 mm chez les Caucasiennes, de 6, Io8 chez les abeilles du Nord ; et de 6,346 chez les Caucasiennes nourries par les abeilles du Nord. D'après les auteurs russes, le mode d'alimentation serait responsable du changement. Peut-être faut-il voir une confirmation de ce point de vue dans les travaux d'Hachinome et OhNishi (I954) suivant lesquels la longueur de la langue ne dépend pas des conditions de nutrition au cours des derniers stades larvaires, mais se trouve fortement influencée au contraire par ces conditions pendant les premiers stades. Alors l'abondance de nourriture: 1a raccourcit, la pénurie l'allonge par rapport aux ouvrières élevées dans les conditions standard. Si bien que tout dépend en définitive de la quantité de gelée royale fournie aux jeunes larves, puisque c'est leur seule nourriture.

Meyírhof ( I957) critique les expériences sur l'influence des conditions d'élevage des larves et leurs conséquences sur la morphologie des adultes. D'après elle, les souches utilisées par les auteurs russes n'étaient pas pures, et l'on ignorait tout en particulier des caractères apportés par le père ; on aurait négligé aussi l'analyse statistique. De plus (critique qui révèle chez son auteur un état d'esprit caractéristique) les modifications obtenues ne sont pas intéressantes, car elles ne persistent pas. En 
réalité, la querelle entre les généticiens classiques à l'esprit trop étroit et les mitchouriniens s'est transportée dans le domaine de la morphologie de l'abeille. Cependant les études sérieuses et approfondies de MichaILOFF ont été effectuées longtemps auparavant et il a utilisé la statistique, contrairement à ce que croit MEYERHOFF.

\section{BIOLOGIE DE L'ABEILLE ADULTE}

\section{Les activités à l'intérieur de la ruche.}

L'évolution des abeilles au cours de leur vie est marquée par toute une série de modifications physiologiques et pondérales. Ainsi, le poids sec et la teneur en azote des têtes et thorax atteignent un maximum à une certaine période de la vie des abeilles puis décroissent, chez les mâles et les reines. Mais poids sec et teneur en azote continuent à augmenter chez les ouvrières jusqu'à la fin de la vie. Mais le poids frais des ouvrières et du thorax des mâles subit une chute brutale dans les premiers jours de la vie. Le taux d'azote de la tête, du thorax et de l'abdomen des ouvrières est assez variable. L a décroissance du poids sec dans la dernière période de la vie doit être attribuée sans doute à la diminution des dépôts de graisse et de glycogène et la décroissance du taux d'azote au début de la vie à la diminution des protéines des tissus et surtout des muscles. (II faut noter que les rats perdent aussi à peu près $30 \mathrm{p}$. roo de leur poids à la fin de leur vie.) Le poids sec et la teneur en azote maxima sont atteints par les ouvrières au bout de 6 jours, au moment où elles deviennent nourrices. La chute initiale du poids frais des très jeunes ouvrières provient sans doute de ce qu'elles ne peuvent encore régler leur teneur en eau. L'augmentation du poids du thorax et de sa teneur en azote dans la dernière période de la vie doit être attribuée à l'activité de vol qui développe sans doute la musculature thoracique. Au contraire, le thorax des reines et des mâles perd du poids à la fin de la vie, sans doute parce que la reine n'utilise pas sa musculature thoracique et que les vieux mâles ne sont guère actifs (HAYDAK, I959).

Une des particularités principales de la ruche consiste dans la régulation thermique. La technique suivie par les ouvrières pour lutter contre l'élévation thermique excessive a été bien étudiée par LINDAUER (I954): comme les guêpes, les abeilles apportent de l'eau sur les rayons et la répandent à leur surface en ventilant activement. I es abeilles peuvent aussi étaler sur la cire des gouttelettes de nectar qu'elles dégorgent, ceci sans doute plutôt dans le but de concentrer le nectar que de régler la température. Il existe des groupes de travailleurs séparés et définis pour l'approvisionnement en eau, pour sa répartition sur les rayons et aussi pour le dégorgement des gouttelettes de nectar. On peut montrer un 
rapport précis entre le degré de surchauffe de la ruche et l'augmentation des apports d'eau. Il est d'ailleurs évident qu'une partie des besoins en eau dépend d'autres facteurs, par exemple de la présence de couvain ouvert. Ajoutons que lorsque la miellée bat son plein, les abeilles ne vont pas chercher d'eau. Il existe d'autre part une certaine compensation des besoins ; quand la pluie ou le froid empêchent pendant plusieurs jours les abeilles de sortir, un plus grand nombre ira puiser de l'eau à la première éclaircie.

LINDAUER (I955) a compté jusqu'à Ioo chercheuses d'eau par jour dans une colonie italienne au cours d'une journée chaude. Il semble que certaines ouvrières particulièrement assoiffées qui quémandent de l'eau avec insistance décident les pourvoyeuses spécialisées à s'envoler pour en puiser ; mais si au contraire les ouvrières n'acceptent de l'eau qu'avec lenteur et réticence, les pourvoyeuses cessent rapidement leur office. Il est assez curieux de noter qu'il ne sort d'abord qu'une seule abeille chercheuse d'eau, qui alertera ensuite toutes les autres par ses danses. Et pourtant, plusieurs doivent être affectées en même temps par la hausse de concentration du contenu stomacal? Mais les échanges doivent être très actifs, car la concentration en sucre du jabot des abeilles butineuses et des abeilles de l'intérieur ne montre que des variations tout à fait parallèles.

Les besoins en eau sont considérables surtout quand la température s'élève. On le voit bien lorsque le trou de vol est obturé par une toile métallique à l'occasion d'un transport ou d'un traitement insecticide. La mortalité est alors très forte, tout au moins en pleine période d'activité, à moins qu'on ne fournisse très libéralement de l'eau à raison d'un litre ou davantage par jour. Alors la température interne baisse brusquement, l'eau est absorbée, et la mortalité reste très faible. L'importance des besoins en eau paraît se concilier difficilement avec le petit nombre des pourvoyeuses d'eau (VUILLAUME, I957).

D'autre part, une activité essentielle dont des travaux de plus en plus nombreux montrent l'extrême importance, consiste dans l'échange mutuel de nourriture. La "demande de nourriture " s'effectue par l'intermédiaire d'une posture spéciale, où la tête de l'abeille fournit tous les stimuli ; une tête isolée peut d'ailleurs suffire à déclencher cette posture. La reine et les mâles peuvent demander de la nourriture, mais d'habitude seules les ouvrières en donnent. Les antennes s'agitent fébrilement pendant la distribution et il est possible qu'il s'agisse d'un mode spécial de communication. Les abeilles peuvent même demander de la nourriture à un leurre formé de petites boules de coton frottées contre la tête des ouvrières, dont sans doute elles prennent ainsi l'odeur; et l'expérience réussit mieux encore si la "tête " porte deux petites " antennes" en fil de fer. Les mouvements de demande de la nourriture à l'aide des antennes 
sont innés mais paraissent manquer de précision chez les jeunes abeilles ; l'abeille sans antennes peut encore offrir de la nourriture, mais non en donner. La couleur et la forme de la tête sont sans importance et les abeilles s'adressent à la partie la plus basse de la tête ou du leurre quelle que soit son orientation. I es échanges se font plus aisément entre abeilles d'une même ruche, et les abeilles de tous les âges procèdent à ces échanges (FreE, x956-59). Istomina Tsvetrova a observé sans interruption deux abeilles de 5 jours à 28 jours. Elles ont donné de la nourriture l'une 20 fois et l'autre Io fois; et demandé, puis reçu, l'une I6 fois, l'autre 8 fois. Il semble que jusqu'à I 2 jours les abeilles reçoivent plus souvent de la nourriture qu'elles n'en donnent mais ensuite ce serait plutôt l'inverse. FREE, a trouvé que la nourriture était plus fréquemment transférée entre abeilles du même âge. La reine est nourrie par les jeunes abeilles seulement et si elle s'égare dans les parties de la ruche où ne se trouvent que les butineuses, celles-ci ne lui offrent rien. A vrai dire, quand elle ne pond pas, la reine peut prélever du miel par elle-même,comme le font d'ailleurs les reines vierges. Il arrive qu'une reine étrangère introduite dans la colonie adopte une attitude de soumission et offre de la nourriture aux ouvrières. Les mâles sont au début nourris entièrement par les ouvrières, mais au bout de 8 jours, ils peuvent prélever eux-mêmes du miel dans des cellules; ils en prennent même beaucoup avant de s'envoler. A l'aide des radioisotopes, Nixon et Ribbands ont montré que les ouvrières donnaient très peu de miel aux mâles (FrEx:, I959). L'échange de nourriture peut s'opérer entre ouvrières même à travers des tôles perforées, à condition que les orifices permettent le contact antennaire; il faut alors pour des trous ronds un diamètre de $3,6 \mathrm{~mm}$, et pour des carrés, 3,2 de côté; pour des fentes 2,8 mm de large (FREE et BUTLER, I958).

\section{La construction.}

Comme je le disais au début il ne peut être question ici de résumer certains travaux particulièrement étendus, comme la thèse de DARCHEN sur la construction de la cire, qui est probablement le travail le plus important paru sur ce sujet depuis HUBER. Quelques points toutefois peuvent être très brièvement évoqués. Ainsi nous retrouvons dans la construction la conception du nombre limite que j'ai exposée ailleurs (Chauvin, I950). Une cinquantaine d'abeilles âgées de six jours peuvent construire un début de rayons avec alvéoles, si une reine vierge ou fécondée se trouve présente. Ce nombre minimum de bâtisseuses s'élève à 200 avec une reine morte et à Io o0o sans reine; avec des ouvrières pondeuses, 5 ooo abeilles suffisent (DARCHEN, I957). Les abeilles réparent très rapidement les trous et fentes pratiqués dans la paroi ou dans le fond des cellules; elles éprouvent plus de difficultés à réparer les cellules 
écrasées par une pression normale à la cloison médiane. Mais ce sont les caractères du fond qui règlent toute la forme de la cellule : à l'aide de certains artifices, on peut poser des cellules sans fond sur un fond différent du leur : fond de mâles pour des cellules d'ouvrières et vice versa. Alors les cellules sont modifiées et partiellement reconstruites pour les " mettre d'accord " avec le fond; c'est-à-dire que les cellules de mâles sont progressivement et plus ou moins irrégulièrement rétrécies pour les ramener aux dimensions d'une cellule d'ouvrière (DARCHEN, I958).

\section{Le butinage.}

Je me contenterai là aussi de noter quelques points de détail en attendant la revue beaucoup plus étendue que I,ECOMTE doit consacrer à ce même sujet dans ces mêmes colonnes.

Les Russes ont trouvé une intéressante modification de l'osmoguidage, que l'on pourrait appeler l'osmoguidage inversé. D'après VoskresSENSKHAYA, IOBASHEY et LORATINA, il suffit de donner aux abeilles une macération des fleurs dont on veut les éloigner après l'avoir additionnée de chlorure de calcium. Et ensuite seulement on leur distribue une macération dans le sirop des fleurs vers lesquelles on veut les attirer.

\section{La récolte de la propolis.}

MEYER (I956) n'a pu observer des abeilles en train de récolter de la propolis sur des bourgeons, mais il 1'a fait récolter après en avoir prélevé dans une ruche et déposé sur une assiette. Dans la région de Berlin, la collecte n'en est opérée qu'en automne, de fin juin à septembre ou même plus tard. Les heures les plus propices sont situées entre ro et I 5 heures, de 8 à 20 heures parfois dans les journées les plus chaudes ; mais la récolte peut continuer par temps couvert ou même par grand vent. I a propolis est tassée dans les corbicules, mais si on l'offre sur la planche de vol, elle est emportée dans les mandibules. Il suffit qu'elle soit présentée à $\mathrm{I}, 5 \mathrm{~cm}$ de la planche de vol pour que l'abeille prenne la peine de la tasser dans les corbeilles; ce tassement s'opère au sol et non au vol comme pour le pollen. L'abeille arrache la résine avec les mandibules, la manipule avec les deux pattes antérieures, passe à une des pattes médianes qui la transfère aux corbicules du même côté. Auparavant les antennes tâtent la résine avec minutie comme si elles recherchaient un point d'attaque. L'abeille ne paraît nullement gênée par le caractère gluant de la propolis sans doute à cause de la sécrétion des glandes mandibulaires, qui sert de lubrifiant. En pleine récolte les abeilles s'interrompent et retournent à la ruche où elles restent un moment, et recommencent fréquemment ce manège. MEYER suppose qu'elles vont s'alimenter, car la propolis ne 
renferme aucune substance nutritive. A la ruche d'autres abeilles détachent la charge des récolteuses, particule par particule, ce qui peut prendre plusieurs heures pendant lesquelles la récolteuse reste inactive. La résine est collée ensuite là où il convient, mais très souvent après avoir été mélangée de cire; dans certains cas, la cire peut même remplacer totalement la propolis. L'inverse aussi peut se produire, et il arrive que dans beaucoup de petits travaux (Kleinbauarbeiten) par exemple dans l'operculation des cellules, la cire soit remplacée par de la propolis. Parmi les travailleuses de la propolis on en trouve d'occasionnelles, qui ne se livrent à cette activité qu'entre d'autres travaux ; mais il existe aussi des abeilles à propolis "sensu stricto". On peut cependant détourner vers la récolte du miel des abeilles à propolis; mais elles ne rapporteront jamais ni eau ni pollen. Les propoliseuses se recrutent d'habitude chez les vieilles butineuses à glandes cirières complètement atrophiées ; deux seulement, sur 20 prélevées au hasard par MEYER, possédaient encore des glandes fonctionnelles. Leur nombre est toujours très petit. On en a vu danser, mais on ne peut décider encore si leurs danses alertent réellement les autres abeilles.

\section{L'essaimage.}

Le problème de l'essaimage lui aussi est plein d'obscurité. Sa fréquence varie d'année en année et d'emplacement en emplacement. Il se produit surtout par temps chaud et miellée abondante mais spécialement dans la première moitié de l'été. Au cours d'une enquête poursuivie en Écosse de 1949 à 195 I et qui a porté sur 280 ruches, on trouve que le temps maximum des essaimages coïncide avec la première semaine de juillet, mais beaucoup d'essaimages se situent entre le 25 mai et le 30 juillet. Il n'y a pas de rapport avec le type de ruche, son volume, la force de la colonie au printemps, l'âge de la reine, le nourrissement de printemps, la race, l'usage de la grille à reine, les maladies de l'abeille adulte, la date d'apparition des mâles adultes et des cellules royales. Mais on voit une corrélation positive avec les cadres très rapprochés et le clippage des reines, une corrélation négative avec une forte proportion de vieux cadres au printemps (MURRAY et JEFFREE, I955). L'augmentation du nombre des nourrices n'est pas une cause obligatoire de l'essaimage. Il semble, mais ce n'est pas démontré, qu'une déficience d'ectohormone provoque l'essaimage quand les circonstances extérieures sont favorables. La longueur du jour n'intervient pas non plus, puisque le rapport de ce facteur à l'élevage dans les pays tropicaux est l'inverse de celui des pays tempérés. L,e rapport abeilles/couvain ne gouverne pas non plus l'élevage des reines en vue de l'essaimage (Simpson, I959). (V. plus haut, travaux d'UChida et SAKagami).

D'autre part, les essais de sélection de souches non-essaimantes 
n'ont jamais donné IOO p. IOO de résultats et WEDMORE affirme même que dans les colonies ainsi sélectionnées les abeilles ne remplacent pas volontiers leur reine en cas d'accident. Il est bon, semble-t-il de fournir de l'espace aux colonies à un certain moment optimum et avec des cadres bâtis, car souvent l'indigence de la miellée ne permet pas de bâtir rapidement les cires gaufrées. Il est vrai que Wulfratr de Mexico prétend au contraire réduire l'essaimage en remplaçant chaque année la moitié des cadres par des cires gaufrées! Herrod Hempsali signale que lorsqu' on pose la hausse après que les abeilles ont commencé à étirer la partie supérieure des cadres pour y mettre du miel, il arrive que toutes les ouvrières s'assemblent en quelques heures dans la hausse. Plusieurs auteurs écrivent aussi que l'essaimage paraît moindre dans les ruches dites horizontales (type Layens) que dans les verticales et cela se confirmerait d'après les recherches de Murray et JEFFreE (I955). Un bon moyen de réduire l'essaimage consisterait à remplacer les reines par d'autres élevées dans l'année même, car les reines très jeunes paraissent s'opposer à l'essaimage. L'enlèvement des cellules royales ne l'empêche pas forcément et les méthodes habituelles de prévention, comme le plan Demaree, quoique assez efficaces, ne sont pas non plus assurées du succès dans tous les cas (SrMpson, 1958).

Il est évident d'autre part qu'on ne saurait comprendre le mécanisme de l'essaimage si on n'arrive pas à le déclencher. Mais c'est extrêmement difficile et Simpson n'y est parvenu que par le surpeuplement et l'enlèvement des œufs et seulement dans un petit nombre de cas (Simpson, I957).

ALLEN (I956) a suivi avec précision les altérations de comportement qui accompagnent l'essaimage. Sur une ruche à trois cadres, une première cellule royale fut bâtie le 28 mai et $I 7$ autres entre le 9 et le 14 juin. Des reines vierges en sortirent. $L_{\text {a }}$ reine continua à pondre jusqu'au jour de l'essaimage; on l'a même vue cinq fois pondre dans des cellules royales ou tout au moins prendre la posture de ponte. Elle examine fréquemment les cellules royales et les ouvrières ne l'en empêchent que rarement, elles la laissent même les démolir à moitié lorsqu'elles sont operculées, bien que cela semble les "énerver " beaucoup. Mais dès que les cellules commencent à se former, la reine est nourrie moins souvent, et même il arrive fréquemment que les abeilles lui refusent de la nourriture. Puis la reine est repoussée de part et d'autre et peut même se faire emballer de façon transitoire. I,es abeilles" la secouent énergiquement et la reine peut alors émettre un cri spécial qui semble " pétrifier " celles qui la molestent tant qu'il dure. Avant l'essaimage on observe des colonnes d'abeilles stationnant sur le côté des cadres. Reines vierges et reine fécondées résistent désespérément aux ouvrières qui veulent les pousser dehors et ne se décident qu'à la fin. 


\section{L'hivernage.}

L'entrée en hivernage est assez variable; les reines d'un an pondent Io jours plus tard en automne que les reines de deux ans et $\mathrm{r} 7$ jours plus tard que les reines de trois ans. Des colonies pourvues d'une reine de un an manifestent moins de mortalité au printemps. D'ailleurs la perte des reines en hiver dépend aussi de leur âge. On compte 0,3 p. Ioo de perte pour les reines d'un an; $2,9 \mathrm{p}$. Ioo pour celles de deux et Io p. Ioo parmi celles de trois ans. $\mathrm{L}_{\text {a }}$ mortalité serait plus forte parmi les reines provenant d'un élevage en cupules artificielles que parmi celles que les abeilles ont élevées spontanément (NESTERovodSkII, I953). HaLlund (I956) a étudié complètement la répartition des abeilles dans la grappe en hivernage ; pour cela il a tué une colonie en janvier à l'aide d'acide cyanhydrique et a examiné les ouvrières une par une. 4345 se trouvaient sur les rayons, I I49 dans les cellules, $25^{\circ}$ au-dessus des cadres, 300 sur les bords des cadres. Les abeilles plus jeunes, d'une teinte plus claires se rencontrent un peu partout mélangées aux abeilles plus foncées. Le rectum des abeilles de la périphérie est beaucoup moins plein qu'au centre ; mais beaucoup d'abeilles du centre n'ont guère de miel dans l'estomac; celles de la périphérie en ont davantage et il est plus concentré. La colonie en question avait consommé II9 g par jour, soit 3,I5 $\mathrm{mg}$ par abeille ; ou encore, 20 abeilles consomment $40 \mathrm{mg}$ à l'heure. Elles commencent par ne percer qu'un petit trou dans l'opercule des cellules à miel et ne l'enlèvent complètement que lorsqu'elles ne peuvent plus atteindre le fond. D'autre part la concentration en gaz carbonique de l'atmosphère interne s'accroît temporairement à la suite du plus léger trouble, par exemple un simple grattage de la ruche. Le taux en gaz carbonique est un peu plus élevé au centre de la grappe qu'à la périphérie, plus haut dans les ruches les mieux isolées thermiquement et dans les jours les plus froids. Les abeilles tolèrent d'ailleurs $9 \mathrm{p}$. IoO de $\mathrm{CO}_{2}$, mais ventilent toujours à partir de Io p. Ioo. I a concentration est plus forte à bâtisses chaudes qu'à bâtisses froides (HALlund, I956).

\section{LE COMPORTEMENT ET LES ORGANES DES SENS}

\section{Réflexes.}

Dans la ligne pavlovienne, les Russes ont effectué d'assez intéressantes recherches sur les réflexes des abeilles. VoskrEsSEnskaya et Lopatina (I952) les habituent à venir sur des tablettes colorées et parfumées; les ouvrières se dressent plus facilement à une odeur qu'à une couleur (odeur de trèfle). Un signe complexe (bleu + alcool octylique) est plus facilement appris que blanc + alcool octylique ou bleu seul. Mais le dressage à un signal complexe est oublié en un jour si l'on cesse de l'accom- 
pagner d'une distribution de miel et les abeilles ne s'intéressent plus alors qu'à une des composantes (bleu seul ou alcool octylique seul). Il est vrai qu'il suffit alors d'une ou deux distributions de miel sur le signal complexe pour qu'il regagne aussitôt toute son efficacité. Il est possible d'effectuer un transfert butyl-bleu sur octyl-bleu après une journée de dressage.

L'hydroxycitronellal, stimulus indifférent, peut provoquer à lui seul l'extension du proboscis si on l'a préalablement associé au sucrose (sur les tarses). L'extinction survient au bout d'une dizaine d'essais sans renforcement. On peut d'ailleurs mettre en évidence comme chez les Mammifères des phénomènes d'extinction temporaire suivis de récupération le jour suivant. La généralisation peut être obtenue sur l'aldéhyde p-isopropyl- $\alpha$-méthylhydroxycoumarique, dont l'odeur est analogue, mais non avec le citral. La généralisation une fois obtenue, on peut obtenir une différenciation en renforçant une des substances et non 1'autre; mais la différenciation ne persiste pas plus d'un jour (TAKE,DA, I957).

On peut aussi, suivant une technique classique, présenter à l'abeille un signal lumineux alors qu' on lui inflige une secousse électrique. Aussitôt l'abeille sort son aiguillon; et le sort au bout d'un certain temps en présence du signal lumineux seul. De même, elle ventile en réponse aux élévations thermiques, qu'on peut associer aussi à un signal lumineux ; au bout d'un temps raisonnable ce dernier déclenchera seul le comportement de ventilation (Lobashisv, 1958). Enfin l'extension de la trompe induite par le trempage des tarses dans une solution sucrée peut aussi s'associer à un signal lumineux d'une certaine intensité et longueur d'onde ; cela est compliqué, car les abeilles allongent le proboscis même avant que la baguette humectée d'eau sucrée ne touche leurs tarses, sans doute parce qu'elles sentent la vapeur d'eau à distance; en effet si la baguette a été seulement trempée dans l'eau et non plus dans une solution sucrée, le même phénomène survient dès qu'on l'approche des tarses. Mais il faut que les antennes soient intactes, si bien qu'elles déterminent probablement à elles seules la réaction. Chez les abeilles sans antennes il est possible alors d'associer lumière et extension de la trompe, et l'on voit alors que les abeilles peuvent différencier 3 régions du spectre, mais sans leur percevoir de limites précises (KUWABARA, I957).

En étudiant l'antenne de l'abeille, Kuwabara et TAKEDA (I956) ont dressé la carte de la répartition des différents sensilla. On peut mesurer alors l'extension du proboscis en réponse à diverses stimulations, mais en l'absence de divers segments antennaires. Chez les animaux ainsi traités dont on stimule les antennes par de la vapeur d'eau, l'extension du proboscis apparait encore quand on a amputé tellement d'articles aux antennes que des animaux neufs, avec aussi peu d'articles, ne pourraient plus développer le réflexe ; chez les animaux neufs, il ne faut pas amputer plus de deux articles et demi pour conserver la réaction. 


\section{L'activité de vol.}

Le nombre des abeilles en vol augmente avec la miellée; mais les petites colonies envoient jusqu'à 50-60 p. Ioo de leur population totale au dehors, même pour une petite miellée ; elles ne peuvent donc augmenter encore leur activité quand la miellée devient plus forte, puisqu'elles font déjà le maximum pour une petite miellée. Seules, les fortes colonies gardent toujours une réserve qu'elles n'emploient que pour une très forte miellée. Elles amassent alors plus de nectar par rapport au poids des abeilles que les petites colonies. Les abeilles des colonies faibles ont aussi une vie plus courte, commencent plus tôt à butiner et volent plus longtemps (TARANOv, I952). Si on enlève la reine le nombre d'abeilles qui prennent leur vol diminue de $77 \mathrm{p}$. I0o, le nectar transporté dans l'estomac de $62 \mathrm{p}$. Ioo, le nombre des abeilles retournant avec des pelotes de $73 \mathrm{p}$. I00, le poids des pelotes de $5^{\circ} \mathrm{p}$. I00, l'accroissement journalier du poids de la ruchede $8 \mathrm{I}$ p. Ioo et la rapidité d'étirage de la cire de $73 \mathrm{p}$. Ioo. Les mesures. ont été effectuées 3 jours avant l'orphelinage et 3 jours après. L'activité est minimum au moment de la fondation des cellules royales et revient peu à peu à la normale par la suite (GENRIKH, I957). Sans doute intervient-il encore des régulations plus compliquées; par exemple TusHmalova (1956) distingue entre les abeilles qui sortent sans hésitation et celles qui hésitent. Il existe aussi de grosses différences individuelles. dans la longueur de la période de vol, et dans le rapport entre l'âge de l'abeille et la durée de son vol.

Il est évident que des facteurs extérieurs gouvernent l'activité de vol, suivant des lois assez mal connues d'ailleurs. Parmi ces facteurs, un des plus importants est la température (mais les rayons solaires jouent aussi un grand rôle). L,e vol commence à des températures variées qui dépendent du minimum nocturne, et si la température matinale ne s'élève. pas au-dessus de ce minimum, elles ne volent pas ; toutefois, de novembre. à février, une faible élévation au-dessus de ce minimum suffit à déclencher le vol; elle doit être plus forte de mars aux mois suivants; puis elle diminue pendant les mois de mai, juin, juillet et août. Pendant le mois de septembre où aucune miellée n'attire les abeilles, la différence minimum. nécessaire par rapport au minimum nocturne augmente jusqu'en octobre Toutefois, on observe des fluctuations d'année en année et de mois en mois dues aux fluctuations de la température de l'air ou à l'acclimatation des abeilles aux conditions de l'environnement pendant les périodes prolongées d'inactivité, etc. A Rothamsted, la durée de vol varie de $2,56 \mathrm{~h}$ en décembre à $\mathrm{I}, 94 \mathrm{~h}$ en juillet. Des colonies de même force ont une activité comparable (WAFA, I955). (I1 y aurait beaucoup à redire sur cette dernière affirmation de WAFA ; des colonies de même force présentent au contraire souvent des différences inexplicables d'activité.). 


\section{LES ORGANES DES SENS}

\section{La vision.}

Je ne puis que rappeler très sommairement ici les immenses recherches de l'école de von Frisch sur la vision des abeilles, que j'ai résumées dans mon traité de Physiologie de l'Insecte (Chauvin, I956). Cependant les travaux particulièrement rigoureux et très récents (1956) de DAUMER nécessitent une mention spéciale. Au lieu d'employer pour le dressage des panneaux colorés dont l'analyse chromatique n'est pas sans difficultés, il se sert de faisceaux monochromatiques purs qui peuvent éclairer un abreuvoir de quartz où l'abeille vient prendre le sirop. Quatre abreuvoirs similaires peuvent ainsi recevoir des mélanges, en proportion connue, de lumière blanche, de radiations colorées et d'ultra-violet. L'auteur commence par mesurer le pouvoir excitateur des radiations par l'appréciation du seuil où elles commencent à agir : l'ultra-violet à $360 \mathrm{~m} \mu$ serait le plus efficace suivi par le bleu-violet à $440 \mathrm{~m} \mu$, le vert à $530 \mathrm{~m} \mu$, le bleu-vert à $490 \mathrm{~m} \mu$ et l'orangé à $6 \mathrm{I} 6 \mathrm{~m} \mu$. On apprécie ensuite la saturation subjective pour l'abeille par la quantité de radiations colorées qu'il faut mêler à la lumière blanche pour que l'abeille reconnaisse l'ensemble pour coloré : l'UV est le plus saturé, puis le bleu-violet et les couleurs de la zone jaune ; le bleu-vert est le moins saturé. La clarté relative ne se déduit pas facilement de la saturation et du pouvoir excitateur : 1'UV paraîtrait le plus clair. Quant au pouvoir de discrimination entre les différentes longueurs d'onde, on voit que dans la zone des jaunes l'abeille distingue l'orangé $6 \mathrm{I} 6 \mathrm{~m} \mu$ du jaune $588 \mathrm{~m} \mu$ et du vert $530 \mathrm{~m} \mu$; dans le vert, le vert $530 \mathrm{~m} \mu$ et le bleu $474 \mathrm{~m} \mu$; dans le bleu, le blew $474 \mathrm{~m} \mu$ et le bleu-violet sont bien mieux distingués que les couleurs de la zone des jaunes; dans 1'ultraviolet, la discrimination s'opère entre l'UV 375 et 1'UV $360 \mathrm{~m} \mu$. Mais il existe une couleur spéciale, le pourpre d'abeille résultant du mélange du jaune et de l'UV. Dans le domaine des pourpres (jaune $588+$ UV 360) on a de 2 à 5 nuances de coloration suivant les individus. On retrouve dans ce mélange la grande saturation de 1'UV puisqu'il suffit de mélanger 2 p. Ioo d'UV au jaune pour que l'abeille le distingue du jaune pur. Le violet d'abeille résulte du mélange bleu-violet + UV. Dans le mélange blenviolet $440+\mathrm{UV} 360$, se distinguent de 3 à 7 nuances suivant les individus; et il suffit que $3 \mathrm{p}$. Ioo d'UV soit mêlé au bleu pour que l'abeille le reconnaisse. Comme le disent HERTz et ENGLANDER, le blanc sans UV agit sur les abeilles comme le bleu-vert non saturé ; le bleu-vert et l'UV sont complémentaires. Le blanc d'abeille se confond avec 85 p. Ioo de bleu-vert et I 5 p. Ioo d'UV. Mais jaune et bleu ne sont pas complémentaires pour l'abeille, et se rapprochent plutôt du blanc sans UV. Le jaune d'abeille est complémentaire du violet d'abeille; le bleu d'abeille complémentaire 
du pourpre d'abeille. En mélangeant le jaune avec le bleu, on obtient le bleu-vert et pour avoir du blanc, il faut alors ajouter de 1'UV. L'abeille percevrait donc six qualités de couleur : jaune, bleu vert, bleu, ultra-violet, pourpre et violet d'abeille. On peut les ramener à trois : jaune, bleu et UV. Le système de perception des couleurs serait donc trichromatique comme chez l'homme et la mouche Calliphora.

La tête des abeilles contient un pigment visuel, le rétinène dont une partie est liée à une protéine pour former une substance photosensible avec maximum d'absorption à $440 \mathrm{~m} \mu$ : or justement un des maxima de sensibilité spectrale des yeux composés et des ocelles se trouve à $440 m \mu$. Toutefois, l'œil de l'ouvrière comporte deux types de photorécepteurs à deux maxima 335-340 $\mathrm{m} \mu$ et à $490 \mathrm{~m} \mu$; il doit donc exister plusieurs pigments visuels (GoldDsMith, I958).

La fréquence de fusion de l'œil d'abeille peut être obtenue en mesurant les potentiels d'action de la rétine en fonction de l'intensité lumineuse. Cette fréquence est à peu près de 165 par seconde pour une ommatidie isolée; mais pour des surfaces oculaires plus grandes, elle peut monter à 300 par seconde; des mesures par la méthode du réflexe optocinétique corroborent ces chiffres. La fusion n'est donc réalisée que pour des fréquences très élevées, parmi les plus hautes que l'on connaisse parmi les yeux d'insectes. Chez Calliphora par exemple elle est de 250 au maximum. Ces fréquences élevées conviennent particulièrement à des insectes à vol rapide comme l'abeille et Calliphora qui doivent pouvoir en très peu de temps distinguer nettement des objets. Ia fréquence de fusion augmente avec l'intensité lumineuse (AUtrum I950).

\section{L'odorat.}

Les travaux sur l'odorat des abeilles, introduits aussi par les magnifiques recherches de von FRISCH, sont très nombreux. Récemment Schwarz (1955) a repris le sujet au moyen de l'olfactomètre de NEUHAUs et aussi par la méthode du dressage. I'abeille se laisse dresser à toutes sortes de composés chimiques synthétiques qu'elle ne rencontre pas dans la nature ; leur seuil d'action se trouve entre I,9.IO $0^{9}$ et 4,3.I $\mathrm{O}^{11}$ molécules par centimètre cube. On trouve d'ailleurs soit les mêmes seuils pour des substances différentes (alcool phénylpropylique, acétate de phényléthyle) ou au contraire des seuils différents pour des isomères ( $\alpha$ et $\beta$ ionone). Dans la série des éthers éthyliques d'acides gras, on observe une baisse du seuil lorsque les chaînes carbonées s'allongent, avec un minimum en Cı2. Dans la série des acides gras, le maximum de sensibilité se trouve situé en $\mathrm{C}_{4}$ (acide butyrique). I a position du seuil semble liée à la solubilité dans l'eau, les graisses ou les protéines. L'acuité olfactive de l'abeille est assez voisine de celle de l'homme, bien qu'elle soit moins sensible aux 
acides gras et à leurs esters éthyliques, et plus sensible aux odeurs florales. Mais à l'intérieur de séries de corps homogènes, les courbes des seuils sont voisines pour l'homme et l'abeille ; dans la série des acides gras, elles se rapprochent aussi de celles du chien et du rat. Si bien que l'organe olfactif des vertébrés et de l'abeille présente une certaine parenté physiologique. Mais l'odorat de l'abeille paraît plus développé que celui de nombreux autres insectes. Il est curieux de noter, comme l'avait déjà fait von FrISCH que l'abeille peut se dresser positivement à des substances répulsives pour elle ou à des acides gras nauséabonds, mais on ne peut toutefois obtenir de dressages positifs au scatol, au benzoate d'isobutyle, à l'huile de patchouli, au sulfure de carbone (et pourtant l'odeur du scatol très dilué rappelle celle des fleurs). L'abeille est un peu plus sensible que 1'homme aux odeurs florales, mais 40 fois moins sensible à l' $\alpha$-ionone et à l'eugénol ; d'après Fischer (I957) l'homme est cinq fois plus sensible à l'odeur de romarin; mais l'abeille est plus sensible que l'homme à l' "odeur de cire " et à celle de la glande de Nassonof. I'abeille distingue l'essence de jasmin du bromostyrol à des concentrations très voisines, mais tout dépend des antennes et la sensibilité décroît proportionnellement au nombre d'articles que l'on a amputés. Les abeilles sans antennes sont insensibles aux odeurs même fortes. Dostar (1956) coupe des portions croissantes des antennes et calcule la sensibilité en présence d'un composé odorant le nérol $\mathrm{B}$. La décroissance ne suit pas une ligne droite, mais est reliée au nombre de sensilla par une courbe logarithmique qui s'exprime par une loi du type WebirR-Fechner.

Il est important de noter que les influx suscités dans le nerf des antennes par différentes essences agissant sur les organes sensoriels ne sont pas les mêmes en été et en hiver. I a latence, l'amplitude et la durée de la réaction sont différentes chez les abeilles d'été et d'hiver. D'autre part 5 p. Ioo de $\mathrm{CO}_{2}$ dans l'atmosphère facilite beaucoup la réaction; et il faut se rappeler que l'atmosphère de la ruche est justement assez riche en gaz carbonique (BoISTEL, I, ECOMTE, CoRABœUF, I956).

\section{Rôle des odeurs dans la vie de l'abeille. L'odeur du corps.}

STEINHOFF (I948) a déjà montré que différentes odeurs de fleurs ou d'huiles éthérées se maintiennent moins longtemps sur le verre ou la porcelaine, le plomb ou la laine de verre que sur le bois, le papier filtre, la ouate et le corps même de l'abeille. L'odeur tient mieux sur les zones pileuses de 1'abeille que sur celles qui sont dépourvues de poils et mieux sur les abeilles vivantes que sur les mortes. Les odeurs florales persistent plus longtemps sur le corps des abeilles que les autres; et parmi les odeurs florales, celle des fleurs visitées par les abeilles davantage que celle des 
autres fleurs. La persistance des odeurs est aussi bonne sur le tégument des bourdons que sur calui des ouvrières; elle est moins bonne sur la cuticule des coléoptères ou des mouches. La persistance des odeurs est ressentie par les abeilles beaucoup plus longtemps que par l'homme, surtout pour les odeurs florales naturelles. C'est la couche cireuse et grasse de la cuticule qui retient les odeurs; lorsqu'on l'enlève par un solvant des corps gras, la persistance des odeurs est réduite de moitié. Mais si l'on en croit RibBands (I955) il est une odeur à laquelle l'abiille est particulièrement sensible, celle de son propre corps ou du corps de ses congénères, même si elles n'exhibent pas la glande de Nassonof. C'est pour cela que les abeilles manifestent une si forte tendance à revenir là où elles sont déjà allées. Et RIBBANDS reproche à von FrISCH d'avoir insuffisamment éliminé ce facteur de perturbation dans ses expériences de dressage. Il les recommence en changeant très souvent les cupules où l'on dépose le sirop attractif; il peut alors montrer, contrairement à von FRIsch, que l'abeille est bien plus sensible que l'homme à de nombreuses odeurs : elle distingue par exemple des mélanges de I p. Ioo d'acétate de benzyle dans II9 parties de linalol et peut même descendre beaucoup plus bas. On les a dressées à reconnaître le même mélange cette fois dans les proportions de I/359; or von FRISCH trouvait que le seuil était seulement pour le même mélange de $\mathrm{I} / 24$.

Mais les odeurs ont une autre action assez difficile à interpréter : elles excitent fortement les danses des abeilles, lorsqu'on mélange des substances parfumées à la nourriture. Parmi les différentes essences (menthe poivrée, anis étoilé, citronelle, lavande, géranium, bergamote, orange, thym) c'est l'essence de citronelle qui donne les meilleurs résultats. Pour l'odorat humain, le seuil de sensibilité à ces substances se rapproche du seuil de dilution où commencent les danses des abeilles. C'est l'odeur elle-même, et non le goût ou la chimio-réception de contact qui en sont responsables. A trop forte concentration d'ailleurs, les danses sont inhibées (1'optimum est à I g par litre et le seuil inférieur à I g/8 litre). Le bromostyrol et la méthylhepténone inhibent les danses à toutes les concentrations (KASCHEF, 1957).

\section{L'audition.}

D'après les anciens travaux de Hansson (I945) 1'abeille émet une vibration de $250 \mathrm{~Hz}$ environ lorsqu'elle arrive à une source de nectar et de 239 quand elle la quitte ; plus forte est la concentration, plus élevé est le son à l'arrivée, mais non au départ. Au cours de la visite des fleurs, la hauteur du son décroît de $25 \mathrm{I}$ à $228 \mathrm{~Hz}$ par exemple. Les collectrices d'eau et de pollen font entendre le même son que les collectrices de nectar. Les abeilles vivement excitées donnent $285 \mathrm{~Hz}$. Le chant de l'essaim consiste dans la sommation des sons normaux émis par les abeilles, mais 
s'accompagne de l'effet Doppler (changement apparent de la hauteur du son qui suit l'augmentation de la distance par rapport à l'observateur). Les mâles donnent 207 vibrations, les reines vierges 254 , les reines fécondes 224. Le "tut tut" des reines à l'éclosion correspond à $435 \mathrm{~Hz}$ et à 493 après deux jours; le "couac " des reines à 323 ; le chant des ventileuses à 2 I5-7.

La réaction des abeilles à un son musical comme celui d'un accordéon, à l'intérieur de la ruche, consiste dans une immobilisation de 5 secondes environ suivie d'un déplacement lent. La réaction la plus nette s'observe dans la bande des 400-600 Hz. Plus le son est fort, plus la réaction est marquée; elle est supprimée au-dessus de ${ }^{5} 500 \mathrm{~Hz}$ et au-dessous de 20 . On ne peut enregistrer de réaction bien caractéristique à aucun des sons qu'on entend dans la ruche, saut au " tut tut " des reines. A l'air libre, les abeilles ne réagissent à aucun son, et on ne peut les entraîner à en associer un avec la nourriture. I, a reine vierge ne réagit aux sons que si elle se trouve près de leur source. FrINGS et LITTLE (I957) ont eu l'idée d'utiliser l'akinèse des abeilles en présence d'un son d'une fréquence de IOO-I 500 cycles par seconde et d'une intensité suffisante (audible à $250 \mathrm{~m}$ ). On peut alors immobiliser les ouvrières pendant 20 minutes, si bien que l'apiculteur peut les manier à volonté ; la fréquence optima est de 500800 cycles ; l'activité reprend dès qu'on arrête l'émission. Il semble bien que les abeilles n'entendent pas en réalité, mais que le son les impressionne grâce à la vibration du substrat. Il n'y a pas d'accoutumance. Notons que les fréquences les plus efficaces correspondent à celles qu'AuTrum et SCHMIEDLER et signalent conme excitant au maximum l'organe subgénual de l'abeille.

\section{Organes de l'équilibre chez l'abeille.}

Dans la zone des articulations tête-thorax et thorax-abdomen on trouve des organes sensibles à la pesanteur. La tête est suspendue à deux prolongements chitineux de l'épisternum; sa partie inférieure est plus lourde, et la tête a tendance à s'incliner vers l'extérieur. Mais un système musculaire compliqué la maintient en position perpendiculaire à l'axe du corps, et il reçoit des ordres d'un organe nuchal situé sur les prolongements épisternaux et qui enregistre tous les déplacements de la masse céphalique. Quand on sectionne le nerf sensoriel qui parvient à ces organes, le comportement géotactique de l'abeille est désorienté et de même l'indication directionnelle au cours de la danse frétillante; il en est de même lorsque la tête est collée au thorax ou lorsqu'on la surcharge d'un poids supplémentaire ; 1'abeille confond alors le dessus avec le dessous. Il existe aussi un organe $d u$ pétiole, qui peut remplacer l'organe nuchal et vice versa, pourvu que la tête soit intacte et occupe par rapport au thorax une 
position normale. Si soit la tête, soit l'abdomen sont fixés en position anormale, 1'organe laissé intact (organe nuchal ou organe du pétiole) ne peut corriger l'exclusion de l'autre (IINDAUir et NEDEL, I959).

\section{Le sens du temps.}

On sait depuis longtemps qu'il est possible d'amener les abeilles à butiner à heure fixe une coupelle de sirop. RENNER a voulu savoir si 1' " horloge " des abeilles était interne (correspondant à un rythme physiologique) ou externe (influence d'un phénomène cyclique terrestre ou cosmique). Pour cela, il élève pendant une année une colonie en milieu strictement confiné, en dehors de toute influence extérieure connue et sous éclairage artificiel. Alors la quinine qui abaisse les échanges et la thyroxine qui les élève (comme Wirnkir l'a prouvé en 1954) n'altèrent pas le sens du temps, que les tests soient réalisés en milieu confiné ou en plein air. Mais si entre la période de dressage et le test de contrôle on abaisse la température à $4^{\circ}$ (alors que tout se passe d'habitude à $25^{\circ}$ ) les abeilles viennent alors avec un certain retard lors du test de contrôle. Dans une première phase de ses recherches, Renver pensait que les influences cosmiques ne jouaient pas. Car après avoir fait franchir à sa colonie $76^{\circ}, 5$ de longitude la perturbation du dressage restait nulle. Mais de plus récentes recherches (1959) l'amènent à admettre une certaine influence exogène.

\section{La mémoire du gîte.}

Après une transposition de ruche, certaines butineuses gardent la mémoire de l'ancien emplacement pendant une douzaine de jours, c'est-à-dire pratiquement jusqu'à leur mort. Après le déplacement de la ruche, l'oubli peut être induit par greffages de cupules royales dans un groupe d'abeilles orphelines; mais il n'est qu'apparent car dès qu'on enlève 1a cupule toutes les abeilles retoument à l'ancien emplacement (VuILLAUME, I959). Iorsqu'on tourne la ruche de $180^{\circ}$, il est possible de voir les butineuses se comporter suivant leur nombre de deux manières différentes. Quand elles sont en petit nombre, elles retrouvent rapidement le trou de vol. Mais lorsqu'elles sont en grand nombre, elles forment, sur le corps de ruche lui-même une piste qui va de l'emplacement de l'ancien trou de vol vers le nouveau (Iticon'tis, I956).

\section{RÉFÉRENCES BIBLIOGRAPHIUQES}

AIIEN (D. M.) et JEFFREE (E. P.). - The influence of stored pollen and colony size on the brood rearing of honeybees. Ann. Appl. Biol., 44, 649-56, I956.

AlLEN (M. I.). - The behaviour of honeybees preparing to swarm. Brit. J. Anim. Behav., IV, 15-22, 1956. 
ALLEN (M. D.). - Observations on honevbees examining and licking their queen. Brit. J. anim. Behav', VI, 8I-4, I957.

ALLEN (M. D.). - Drone brood in honey bee colonies. J. econ. Entom., 51, $46-8$, I 958.

ALLEN (D. M.). - The occurrence and possible significance of the shaking of honeybee queen by the workers. Anim. Behai., VII, 66-70, I959.

Armbruster (L.). - Versuche und Zahlen zum Bienen Brutgeschäft. Arch. Bienenk, VI, 44-57, I924-25.

Autrum (H.) et Stocker (M.). - Iie Verschmelzungsfrequenzen des Bienenauges. $Z$. Naturforsch., 5, 38-43, I950.

Boistei, (J.), LECOmTe (J.) et Corabriuf (E.). - Quelques aspects de l'étude électrophysiologique des récepteurs sensoriels des antennes d'Hyménoptère. Ins. Soc., III, 25-3I, I956.

BUTLER C. G.). - Queen supersedure and swarming. A Lecture given to the Beekeepers Assoc., I2 p., I958.

CALE (G. H.) et Gowex (J. W.). - Heterosis in the honey bee. Genetics., $41,292-303$, I956.

Chauvin (R.). - Les facteurs qui gouvernent la ponte chez la reine des abeilles. Ins. Soc., III, 499-504, I 956.

Darchen (R.), Vizié (L.) et Vuiritadme (M.). - Sur le déterminisme des constructions de cellules de mâles chez les abeilles. C. R. Acad. Sc., 244, 39I-4, I957.

DARCHEN (R.). - La reine d'Apis mellifica et les constructions cirières. Ins. Soc., IV, 32I-25, I957.

DARChEN (R.). - Construction et reconstruction de la cellule des rayons d'Apis mellifica. Ins. Soc., 357-69, I958.

Darchen (R.). - Les techniques de construction chez Apis mellifica. Thèse Paris, 209 p. (Ann. Sc. Nat. Zool., Iz $2^{\mathrm{C}}$ sér., I), I959.

Darchen (R.) et Vuiliaume (M.). - Déterminisme de la construction des cellules de mâles et des cellules d'ouvrières chez Apis mellifica. Ann. Abeille, 1, 7-9, I958.

Darchen (R.) et Vuiliaume (M.). - Deux types de cellules royales chez A pis mellifera. Ibid., 2, I77-83, I959.

DAUMER (K.). - Reizmetrische Untersuchungen des Farbensehens der Bienen. $Z$. vergl. Physiol., 38, 4I3-78, I956.

Demiaxowicz (A.). - Héritage des caractères parentaux chez les abeilles. Pszczelnicze Zeszyty Naukowe, I, 97-I03, I957.

Deodikar (G. B.), Thakar (C. V.) et Shah (P. N.). - Cytogenetic studies on Indian honeybees. Proc. Ind. Acad. Sc., XLIX, I94-206, I959.

Dosral (B.). - Riechfähigkeit und Zahl der Riechsinneselemente bei der Honigbiane. $Z$. vergl. Phy'siol., 41, I79-203, I958.

Fischer (W.). - Untersuchungen ïber die Riechstärke der Honigbiene. Z. vergl. Physiol., 39, 634-59, I957.

Flanders (E.). - - Origenic-ovisorptive cycle in the economy of the honeybee. Ibid., 85, I 76-7, I 957 .

Flaxiders (E.). - Control of sex in the honeybee. Sci. Monthly, r1, 237-40, I950.

FLANDERS (E.). - Embryonic starvation, an explanation of the defective honey bee egg. J. econ. Entom., 52, I66-7, I959.

FREE (J. B.). - A study of the stimt1li which release the food-begging and offering responses of worker honeybees. Brit. J. Anim. Behav., IT, 94-IOI, I956.

FREE (J. B.). - The transmission of food between worker honeybees. Ibid., $\mathrm{V}, 4 \mathrm{I}-7, \mathrm{Ig} 7 \mathrm{.}$

FREE (J. B.). - The food of adult drone honeybees. I bid., V, 7-II, I957.

FREE (J. B.). - The transfer of food between the adult members of a honeybee community. Bee World, 40, I93-201. I959. 
FREE (J. B.) et BUTLER (C. G.). - The size of apertures through which worker honeybees will feed one another, Ibid., 39, 40-42, I958.

FRINGS (H.) et IITTLE (F.). - Reactions of honey bees in the hive to simple sounds. Science, I25, I22, I957.

FyG (W.). - Merkmale zur Altersbestimmung von Bienenköniginnen. Schweizer. Bienenz., 10, 428-32, I956.

FYG (W.). - Uber die verschiedenen Ursachen der Drohnenbrïtigkeit. Ibid., 80, I54-7; I 87-92, I957.

Genrikh (V. G.). - (vol et présence de la reine). Pschelovodstvo, 34, 8-I2, I957.

Goldomith (T. H.). - The visual system of the honeybee, Ann. N. Y. Acad. Sc., 74, 223-29, I958.

Goldsmith (T. H.). - Ann. Nat. Acad. Sc., 44, 123-26, 1958.

Gubin (A. F.) et Khalifmai (I. A.). - (La colonie d'abeilles unité biologique). Dokl. Akad. Nauk., 16, I28-32, I952.

Hamman (E.). - Wer hat die Initiative bei den Ausflügen der Jungkönigin, die Königin oder die Arbeitsbienen? Ins. Soc., IV, 9I-I06, I957.

Hallund (V.). - (Concentration de la ruche en $\mathrm{CO}_{2}$, hivernage). Nord. Bitidskr., 8, 78-82; I06-II3. I956.

Hachinome. (Y.) et Ohnishi (N.). - (longueur de la langue chez l'abeille). Bull. Nat. Instit. Agric. Sc., G, 8, 9-I6, I954.

HANsson (A.). - Lauterzeugung und Lautauffassungsvermögen der Bienen. Opusc. Entom., Suppl., 6, I-I24, I945.

HAYdak (M. H.). - Changes with age in the appearance of some internal organs of the honeybee. Bee World, 38, I97-207, I957.

HAYdak (M. H.). - Do the nurse bees recognize the sex of the larvae? Science, I27, III3, I958.

HAYDAK (M. H.). - Changes in weight and nitrogen content of honeybees. Bee World, 40, 225-29, I959.

Hoffman (I.). - Die Aufzucht weiblicher Bienenlarven (Apis mellifica) ausserhalb des Volkes. Z. Bienenforsch., 3, I34-6, I956.

Hiтchсоск (J. D.). - Honeybee queens whose eggs all fail to hatch. J. econ. Entom., 49, II-I4, I956.

Ignatovic (V. P.). - (Productivité des abeilles dans des cellules élargies.) Pschelovodstvo, 7, 24-6, I953.

JEFFREE (E. P.). - Observation on the decline and growth of the honeybee colony. J. econ. Entom., 48, 723-6, I955.

JEFFREE (E. P.). - A shaped wire grid for estimation of the quantities of brood and pollen in combs. Bee World, 39, I I5-I8, I958.

KASCHFF (A. H.). - Uber die Einwirkung van Duftstoffen auf die Bienentänze. Z. vergl. Physiol., 39, 562-76, I957.

KhaRCheVA (A. I.). - - Pschelovodtsvo, 34, 32-34, I957.

Komarov (P. M.) et Alpatov (W. W.) - - Beiträge zur Kenntnis der Variabilität der Honigbiene. Arch. Bienenk., 15, II-20, 1934.

Kulzhinskaia (K. P.). - Pschelovodtsvo, 36-37, I955.

Kuwabara (M.) et Takeda (K.). - Physiol. Ecol., \%, I-6, I956.

KUWABARA (M.). - Bildung des bedingtenreflexes von Pavlovstypus bei der Honigbiene, Apis mellifica. J. Fac. Sc. Hokkaido Univ., Ser. VI, Zool., 13, 458-64, I957.

LAVIE (P.). - Action antibiotique de quelques substances provenant de 1'abeille ou de la ruche sur Bacillus lavvae et Bacillus alvei, r959.

LAVIE (P.) et PAIN (J.). - Les rapports entre la substance antibiotique des reines et des ouvrières d'abeille, le développement ovarien et l'ectohormone. C. R. Acad. Sc., 248, 455-57, I587, I959.

Lavie (P.) et PAIN (J.). - Relation entre la substance attractive, le facteur antibiotique et le développement ovarien chez la reine d'Apis mellifica. Ibid., 248, 3753-5, I959. 
LECOMTE (J.). - Formation de pistes par les butineuses d'une ruche ayant subi une rotation de $180^{\circ}$. Z . Bieneforsch., 3, 4 p., I956.

I.eveneits (I. P.). - Pschelovodtsvo I954, 36-8; 33, 53-4, I956; 33, 28-9, I956. I954-6.

LINDAUER (M.). - Die Temperaturregulierung der Bienen bei Stocküberhitzung. Naturwiss., 38, 308-9, I95I.

LINDAUER (M.). - The water economy and temperaturregulation of the honeybee colony. Bee World, 35-36, I955.

LINDAUER (M.). - Temperaturregulierung und Wasserhaushalt im Bienenstaat. Z. vergl. Physiol., 36, 39I-432, I954.

Lindauer (M.) et NEDEI (J. O.). - Ein Schweresinnesorgan der Honigbiene. Z. vergl. Physiol., 42, 334-64, I959.

Lobashev (M. E.). - Pschelovodtsvo, 35, 2 I-25, 1958.

MACKENSEN (O.). - The occurrence of parthenogenetic females in some strain of honeybees. J. econ. Entom., 36, 465, I943.

MACKENSEN (O. $/$. - Effect of carbon dioxide on initial oviposition of artificially inseminated and virgin queen bees. J. econ. Entom., 40, 344, I947.

MACKENSEN (O.). - Viability and sex determination in the honey bee, Apis mellifera. Genetics, 36, 500-9, I95I.

MedvedeV (I. K.). - Pschelovodstvo, 33, IO-I3, I956.

Meyer (W.). - Die Kittharzbiene und ihre Tätigkeiten. Z. Bienenforsch, 2, I85-200, I954.

Meyer (W.). - Propolis bees and their activities. Bee World, 37, I25-36, I 956.

MEYERHOFF (G.). - Andern sich die Rassenmerkmale durch den Einfluss fremder Ammenbienen? Leipziger Bienenz., 71, I57-9, I957.

Michaer, (A. S.) et AbRamovitz (M.). - A method of rearing honeybee larvae in vitro Bee World, 36, I59-I6I, I955.

Michailoff (A. S.). - Der Einfluss einiger Lebensfaktoren auf die Variabilität der Honigbiene. Arch. Bienenk., VIII, 289-304, I927.

Michailoff (A. S.). - Weitere Untersuchungen über die phänotypische Variabilität der Honigbiene (Apis mellifica) in Verbindung mit der Frage der Bienenrassen. Arch. Bienenk., XII, 39-53, I93I.

MrLojeVIC (B. D.). - Uber der Möglichkeit der Bienenköniginnenzncht ausserhalb des Stockes. Schweiz. Bienenzeit, 8, I94r.

Murray (L.) et JefFreE (E. P.). - Swarming in Scotland. Scottish Beekeeper, 31,96, I 955 .

NeSTERovodskir (V. A.). - Pschelovodtsvo, (7), 3-6, I953.

NoLD (R.). - Ein Beitrag zum Bienenflug am Hang und zum Biennflug überhaupt. Die Biene, 92, 74-6, I956.

Opfinger (E.). - Zur Psychlologie der Duftdressuren bei Bienen. Z. vergl. Physiol., 31, 44I-53, I949.

Orosi PaL (Z.). — Méhészet, 5, I23-4; 223-5; 6, I33-4, I958; 6, 7I-3, I957.

PaIn (J.). - Mesure du pouvoir inhibiteur et de l'attractivité de l'ectohormone des reines d'abeilles. C. R. Acad. Sc., 242, I080-2, I956.

PaIN (J.). - Étude de l'apparition de l'attractivité chez les reines vierges d'abeilles. Ibid., 248, 32II-2, I959.

Podoba (E. G.). - Pschelovodstvo, 4, I7-20, I955.

Prost (P. J.). - La séquestration naturelle des jeunes reines d'abeilles. C. R. Acad. Sc., 24\%, 2042-44, I958.

RENNER (M.). - - Neue Versuche über den Zeitsinn der Honigbiene. Z. vergl. Physiol., 40, 85-II8, I957.

Renner (M.). - The scent perception of the honeybee. Proc. Roy. Soc., B, I43, 367-79, I955.

RobINSON (F. A.). - Effect of electric shock on oviposition in queen bees. Amer. Bee J., 91, 508-9, I95I. 
ROTHExBUHLER (W. C.). - Hereditary aspects of gynandromorph occurrence in honeybees (Apis mellifera). Iowa St. Coll. J. Sc., 29, 487-8, I955.

ROTHENBUHLER (W. C.). - Diploid male tissue as new evidence of sex determination in honeybees. J. Heredity, 48, I60-8, I957.

Rutrier (F.). - Die Sexualfunktion der Honigbienen im Dienste ihrer sozialen Gemeinschaft. $Z$. vergl. Physiol., 39, 577-600, 1957.

SAKAGAMI (S. H.). - The false queens fourth adjustive response in dequeened colonies. Behaviour, XIII, 280-97, I958.

Simpson (J.). - - Observations on colonies of honeybees subjected to treatments designed to induce swarming. Proc. Roy. Entom. Soc., 32, I8592, 1957 .

Simpsox (J.). - The factors which cause colonies of Apis mellifica to swarm. Ins. Soc., V, 77-93, I958.

Simpsox (J.). - The problem of swarming in beckeeping practice. Bee World, 39, I93-202, I958.

Simpson (J.). - Variations in the incidence of swarming among colonies of Apis mellifica throughout the summer. Ins. Soc., VI, 85-98, I959.

SCHIAAjeW (A.). - Pschelorodstro, j, I952.

Schinieva. - Sur l'élevage des reines. Pschelovodtsio, I952.

Schwarz (R.). - Uber die Riechschärfe der Honigbiene. Z. z'ergl. Physiol., 37, I $80-210$, I955.

Skrobal (D.). - Trav. Scientif. Inst. Tchécosl. Apic., I5I-64, I958.

Smirnov (I. V.). - Pschelordstvo, 2, 23-25, I953.

SMith (M. V.). - Queen differentiation and the biological testing of the royal jelly. Comell Enir. Agric. Exp. Stat., 356, 3-5(), I959.

Soczer (S.). - Pszc. Zesz. Naurowe, 11, 9I, Io7-20, I958.

STEINHOFF (H.). - Untersuchungen iber die Haltfähigkeit von Iuftstoffen am Bienenkörper. Z. vergl. Phvsiol., 31, 38-57, 1948.

TAKEDA (K.). - Dobutsug. Zasshi, 66, 44I-9, I957.

Taranov (G. F.). - Zool. Zh., 31, 6I-7I, I952.

TUCKER (K. W.). - Automictic parthenogenesis in the honeybee. Genctics, 43, 299-316, 1958.

Tushmalova (N.). - Pschelotodtsio, 33, 26-28, I056.

UCHIDA (T.) et SAKAGAMI (S. F.). - The seasonal population trends of the honey bee in Sapporo, with some remarks on Supersedure. Jap. J. Zool., 11, 55-62, I955.

VAGT (W.). - Morphologische Untersuchungen am Naschaffungsköniginnen von Apis mellifica, die auf verschiedenalten Larven gezüchtet werden. Z. Bienentorsch., 3, 73-80, I955.

Volosevich (A. P.). - Ag. Pschelovodtsvo, 20-22, I955.

Volosevich (A. P.). - Agrobiologie, 2, I39-4I, I958.

Voskressenskhaya (A. K.) et Lopatina (N. G.). - Z. Obsc. Biol., 13,42I$34,1952$.

Voskressenskhaya (A. K.), Lobashev (M. K.) et Lopatina (N. G.). Pschelovodtsio, 2, 45-48, I954.

VuiliaUme (M.). - L'importance de l'approvisionnement en eau dans la ruche. Ins. Soc., IV, 3I-4I, I957.

Vuildaume (M.). - Contribution à la psychophysiologie de l'élevage des reines chez les abeilles. Ibid., II 3-56, I957.

Vurliaume (M.). - La forme des cellules royales chez les abeilles. Ibid., 387-90, I957.

VUildaUme (M.). - Les substances inhibitrices de la construction des cellules royales chez les abeilles. C. $R$. Acad. Sc., 246, I298-9, 1958.

Vuillaume (M.). - La théorie de Butler sur l'ectohormone des abeilles et ses limites. Ibid., I927-9, I958.

Vuillaume (M.). - Essai de modification des réactions instinctives des abeilles au cours de l'élevage royal. Ibid., 2169-7I, $195^{8 .}$ 
Vuiliaume (M.). - Techniques d'élevage des reines. Ann. Abeille, 1, I89-96, I958.

Vuiliaume M.. - Nouvelles données sur la psychophysiologie de l'élevage des reines chez Apis mellifera, Ibid., 11, I959.

Vurliaume (M.). - La rétention mnémonique chez Apis mellifera, Ibid., 2, I59-70, I959.

WAFA (A. K.). - Temperature as a factor controlling the commencement of honeybee flighting and honeybee flight activity distribution during the various months of thenyear. Bull. Soc. Entom. Egypte, 39, 315-33, I955.

WEAVER (N.). - Rearing of honeybee larvae on royal jelly in the laboratory. Science, 121, 509-Io, I955.

WeAver (N.). - Effects of larval age on dimorphic differentiation of the female honeybee. Anm. Entom. Soc. Amer., 50, 283-84, I957. 